\title{
Projections of IoT Applications in Colombia Using 5G Wireless Networks
}

\author{
Alexis Barrios-Ulloa ${ }^{1,2}$, Dora Cama-Pinto ${ }^{3, * \mathbb{D}}$, Johan Mardini-Bovea ${ }^{4}$, Jorge Díaz-Martínez ${ }^{2}$ \\ and Alejandro Cama-Pinto ${ }^{5, *(D)}$
}

1 Department of Electronics Engineering, Faculty of Engineering, Universidad de Sucre, Sincelejo 700001, Colombia; alexis.barrios@unisucre.edu.co or abarrios56@cuc.edu.co

2 Department of Computer Science and Electronics, Universidad de la Costa, Bicentennial Scolarship-Ministry of Sciences, Barranquilla 080002, Colombia; jdiaz5@cuc.edu.co

3 Department of Computer Architecture and Technology, University of Granada, 18071 Granada, Spain

4 Faculty of Engineering, Universidad del Atlántico, Barranquilla 081001, Colombia; johanmardini@uniatlantico.edu.co

5 Department of Computer Science and Electronics, Universidad de la Costa, Barranquilla 080002, Colombia

* Correspondence: doracamapinto@correo.ugr.es (D.C.-P.); acama1@cuc.edu.co (A.C.-P.)

Citation: Barrios-Ulloa, A.;

Cama-Pinto, D.; Mardini-Bovea, J.; Díaz-Martínez, J.; Cama-Pinto, A. Projections of IoT Applications in Colombia Using 5G Wireless

Networks. Sensors 2021, 21, 7167.

https://doi.org/10.3390/s21217167

Academic Editor: Lorenzo Vangelista

Received: 13 August 2021

Accepted: 25 October 2021

Published: 28 October 2021

Publisher's Note: MDPI stays neutral with regard to jurisdictional claims in published maps and institutional affiliations.

Copyright: (C) 2021 by the authors. Licensee MDPI, Basel, Switzerland. This article is an open access article distributed under the terms and conditions of the Creative Commons Attribution (CC BY) license (https:// creativecommons.org/licenses/by/ $4.0 /)$.

\begin{abstract}
Wireless technologies are increasingly relevant in different activities and lines of the economy, as well as in the daily life of people and companies. The advent of fifth generation networks (5G) implies a promising synergy with the Internet of Things (IoT), allowing for more automations in production processes and an increase in the efficiency of information transmission, managing to improve the efficiency in decision-making through tools such as big data and artificial intelligence. This article presents a description of the 5G implementation process in Colombia, as well as a revision of opportunities when combining with IoT in featured sectors of the departmental development plans, such as agriculture, tourism, health, the environment, and industry. Results shows that the startup of $5 \mathrm{G}$ in Colombia has been a slow process, but there are comparisons with similar procedures in other developed countries. Additionally, we present examples of $5 \mathrm{G}$ and IoT applications which can be promoted in Colombia, aimed at improving the quality of life of their habitants and promoting economic development.
\end{abstract}

Keywords: 5G; frequency bands; Colombia; IoT; radioelectric spectrum

\section{Introduction}

The demand of mobile data services is one of the factors that conditions the development of future wireless networks. The 2020 report of Ericsson indicates that the mobile data traffic in that year was 50 Exabytes (EB)/month, and it is estimated that it could be incremented to $150 \mathrm{~EB} / \mathrm{month}$ in 2024 [1]. This increase is associated with a higher need of services by users and requirements of technologies such as Internet of Things (IoT), Wireless Sensor Networks (WSN), and Artificial Intelligence (IA). In order to meet demand, work began in 2012 on a Fifth Generation wireless technology (5G) [2].

The 5G network presents considerable improvements over fourth generation networks (4G), highlighting the increment of the transmission rate, ultra-reliability, less latency, and higher connectivity [3,4]. Some countries (Japan, South Korea, China, United States, Germany) finished test stages and began technology commercialization. Colombia has not been oblivious to this evolution, currently having $5 \mathrm{G}$ networks in pilot test processes before launching its service to the public. In 2020, the Ministry of Information and Communication Technologies (MinICT) issued a resolution, opening a process of granting permissions for the use of the radioelectric spectrum in different frequency bands, with the purpose of starting pilot tests which uses $5 \mathrm{G}$ mobile technologies [5]. Also, the ministry of ICT states that the 5G frequency bands' concession stage will start before September 2022 [6]. 


\subsection{IoT and $5 G$ Networks}

IoT is increasingly involved in different areas, including home automation, health, agriculture, industry, and logistic. The 5G networks exceed the limitations of $4 \mathrm{G}$ in terms of security, low latency, higher data transfer speed, and a higher quantity of connected devices on their communications, allowing for the possibility of a wide implementation of IoT applications [7,8]; many of which have low potency and latency requirements [9]. Therefore, different initiatives worldwide (for example, 4G America, IMT-2020, 5G Forum) have worked on the adoption and standardization of IoT enabled for 5G [10]. Also, the 3rd Generation Partnership Project (3GPP) has included IoT on their standards. For example, in June of 2020, an Industrial IoT (IIoT) work item was initiated and completed as a central element of Release 16, and is oriented to robotic applications and autonomous systems (RAS) [11].

In addition to the industrial field, it is possible to integrate IoT, through $5 \mathrm{G}$, with other sectors, such as home, cities, and farms [9]. In Table 1, a general description of the typical requirements of IoT applications that the fifth generation networks should satisfy is presented.

Table 1. General description of features/typical IoT application requirements.

\begin{tabular}{ccccc}
\hline Application & Aplication Domain & $\begin{array}{c}\text { Tolerable } \\
\text { Delay }\end{array}$ & $\begin{array}{c}\text { Update } \\
\text { Frecuency }\end{array}$ & Data Rate \\
\hline Structural health & Smart city & $30 \mathrm{~min}$ & $10 \mathrm{~min}$ & Low \\
Waste management & Smart city & $30 \mathrm{~min}$ & $\begin{array}{c}\mathrm{h} \\
\text { Low }\end{array}$ \\
$\begin{array}{c}\text { Video Surveillance } \\
\text { Air Quality Monitoring }\end{array}$ & Smart city & Seconds & Real time & High \\
Monitoring and supervision & Smart home & $5 \mathrm{~min}$ & $30 \mathrm{~min}$ & Low \\
Closed loop control & Industrial & Seconds or ms & Seconds & Milliseconds \\
Interlocking and control & Industrial & Milliseconds & Milliseconds & High \\
Patient's healthcare delivery & Healthcare & Low (seconds) & 1 Report per hour day & Requires Ad-hoc \\
and monitoring & & & emergency & High \\
Real-time emergency response & Healthcare & Low (seconds) & & \\
and remote diagnostics & & & &
\end{tabular}

However, despite the advantages of 5G IoT, its implementation will bring a series of challenges that need to be overcome, including scalability, network management, interoperability, and heterogeneity [12].

\subsection{G Application Scenarios}

Networks commercially operating with $5 \mathrm{G}$ are supported with the technology New Radio (NR), specified by the 3GPP in the Release 15, which is largely based on the recommendations given by the International Telecommunications Union (ITU) [13]. Some of the most important technical characteristics are the use of Orthogonal Frequency Division Multiplexing (OFDM) and the operation up to $52.5 \mathrm{GHz}$, through time division duplex (TDD) or frequency division duplex (FDD) [14]. In addition, Release 15 added new bands for 5G: $3300 \mathrm{MHz}-4200 \mathrm{MHz}, 3300 \mathrm{MHz}-3800 \mathrm{MHz}, 4400 \mathrm{MHz}-5000 \mathrm{MHz}$, $24,250 \mathrm{MHz}-27,500 \mathrm{MHz}$, and $37,000 \mathrm{MHz}-40,000 \mathrm{MHz}$ [15]. For the purpose of satisfying the future requirements of the market and especially of the industry, ITU divided 5G into three application scenarios [16]: (a) enhanced mobile broadband (eMBB), focused on a high transmission rate and targeting applications in busy indoor and outdoor environments, as well as a possible last-mile solution in areas where there is an absence of copper or fiber optic wired networks; (b) massive machine-to-machine communications (mMTC), specially designed to support a high density of equipment per square kilometer, such as IoT applications in smart environments, e.g., smart cities, smart farm, smart home, among others; and (c) ultra-reliable low latency communications (uRLLC) with lower transmission 
rates, intended to support industrial automation or applications requiring mobility, such as autonomous vehicles or patient monitoring. Figure 1 presents a summary of the main characteristics of the three categories.

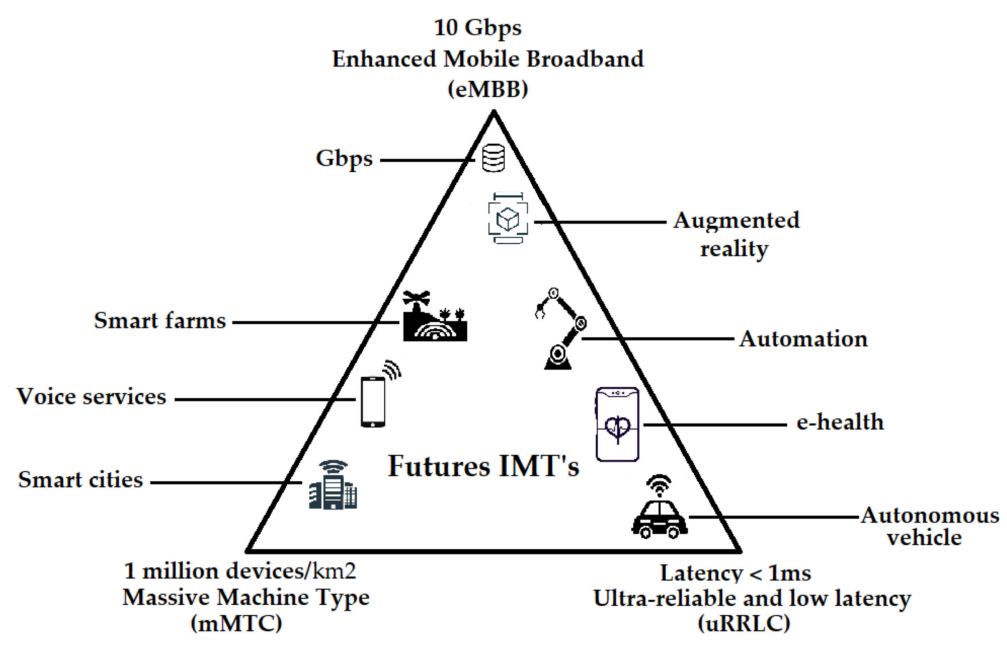

Figure 1. 5G Application Scenarios. Source: $[16,17]$.

Part of the 5G development is based on the fact that some applications will work in frequencies located in much higher bands than the ones used by the current $4 \mathrm{G}$ networks, as is the case with the so-called "high band" spectrum range between $24 \mathrm{GHz}$ and $100 \mathrm{GHz}$ [8]. To this end, the use of tools is required to minimize the problems associated with loss in propagation [18]. For this reason, the implementation of technologies of multiple massive transmission and reception (mMIMO) antennas that use spatial multiplexing to improve the channel capacity is considered, in addition to using large-scale antenna systems, and thus serving the growing number of users [14].

\subsection{Goals and Motivation}

- The main objective of this article is to show the progress for the deployment of the $5 \mathrm{G}$ network in Colombia, as well as the availability of the spectrum and the future needs of this resource according to the considerations established by regulatory agencies. In addition, an analysis of potential IoT applications in different sectors of the Colombian economy that could contribute to the development of the regions with the implementation of $5 \mathrm{G}$ is presented. The specific objectives of this article are:

- Associate IoT applications with 5G in the future demand of the main economic activities of each department and region in Colombia.

- Contextualize the current situation of 5G network deployment in Colombia.

- Relate the 5G frequency bands that will be implemented in Colombia with the most suitable IoT applications for the demands of economic activities in each department and region.

Our work will be a roadmap for future research in the development of IoT applications with 5G, according to the demand of the most relevant economic sectors in different parts of Colombia.

In this research, we present the demand for IoT applications that will be enhanced when working in combination with 5G networks in Colombia. For this purpose, a search was conducted in the Scopus database, selecting articles published as of 2017. We used search strings where the terms " $5 \mathrm{G}$ ", "IoT", "Colombia", "spectrum", "frequency bands", "trends", "challenges", "agriculture", "livestock", "e-health", “tourism", "environment", and "industry" were a part of the titles, abstracts, and keywords. Subsequently, we reviewed each of the documents, focusing on the issues raised, the results, and the conclusions. Additionally, we analyzed different documents from official regulatory organizations, 
manufacturers, operators, and governmental institutions, obtaining a more commercial vision of the future of $5 \mathrm{G}$ in Colombia.

The following criteria were considered in the final selection of the bibliography: a focus on IoT, a focus on IoT/5G, the relation of the subject matter with the Colombian context, the relevant problems of the Colombian economic and social sector, and government information. In total, we selected and used 193 references, of which 69 correspond to journal articles or conferences, 10 to private companies or organizations, 12 to regulators or foreign entities (external to Colombia), and 102 to Colombian public sector entities.

The structure of this article is as follows: Section 2 describes an analysis of the results and provides examples with the use of IoT and 5G in different sectors of Colombia. Finally, Section 3 shows the discussion and conclusions.

\section{Results and Analysis}

\subsection{Projected Use of 5G Frequency Bands}

Current saturation of the radioelectric spectrum has highlighted that, in different countries, the new auctions should principally be between 1 and $6 \mathrm{GHz}$. However, the growing need for a bandwidth that supports $5 \mathrm{G}$ services has brought into consideration portions of the spectrum that were previously not widely accessed; for example, the sub-GHz and the millimetric (mm-W). In Table 2, we highlight some examples of bands auctioned in some countries and their expectations about the allocation of 5G spectrum in the future.

Table 2. Examples of auctioned bands for 5G service provision. Source: own elaboration.

\begin{tabular}{|c|c|c|c|}
\hline Country & Bands Auctioned & Expectation & References \\
\hline USA & $\begin{array}{c}\text { 3.7 GHz, } 24 \mathrm{GHz}, 28 \mathrm{GHz}, 37 \mathrm{GHz}, \\
39 \mathrm{GHz}, 47 \mathrm{GHz}\end{array}$ & Auction the $2.5 \mathrm{GHz}$ and $3.45 \mathrm{GHz}$ bands & {$[19,20]$} \\
\hline Spain & $3.6 \mathrm{GHz}-3.8 \mathrm{GHz}$ & Auction the $700 \mathrm{MHz}$ and $26 \mathrm{GHz}$ bands & [21] \\
\hline Greece & $700 \mathrm{MHz}, 2 \mathrm{GHz}, 3.4-3.8 \mathrm{GHz}, 26 \mathrm{GHz}$ & $\begin{array}{l}\text { Public inquiries for the } 1.5 \mathrm{GHz}, 2.1 \mathrm{GHz} \text {, } \\
\qquad 2.3 \mathrm{GHz} \text { bands }\end{array}$ & [22] \\
\hline Sweden & $2.3 \mathrm{GHz}, 3.5 \mathrm{GHz}$ & Have the band from $3.72 \mathrm{GHZ}$ to $3.8 \mathrm{GHz}$ & [23] \\
\hline United Kingdom & Not assigned & Auction $700 \mathrm{MHz}$ and 3.6 to $3.8 \mathrm{GHz}$ bands & {$[24]$} \\
\hline Japan & $3.7 \mathrm{GHz}, 4.5 \mathrm{GHz}, 28 \mathrm{GHz}$ & Assignment of the $28.3 \mathrm{GHz}$ and $29.1 \mathrm{GHz}$ bands & [25] \\
\hline
\end{tabular}

In the Colombian case, to cover future needs and in harmony with the provisions of the 2019 World Radiocommunication Conference (WRC-19) [13], there is a need to arrange the spectrum in three (03) types of bands of frequency: (a) lower than $1 \mathrm{GHz}$, (b) between 1 and $6 \mathrm{GHz}$, and (c) higher than $6 \mathrm{GHz}$. In this sense, the $5 \mathrm{G}$ plan developed by MinICT has identified a set of candidate frequencies to be used in future networks [26], which are presented in Table 3.

All of these bands have not been attributed to the mobile service provision according to the presented data on the National Table of Attribution of Frequency Bands [27], which will force entities to perform modifications in the future, or perform studies that allow for an evaluation of their compatibility with other already attributed services. Actually, there is not a formal definition of the $5 \mathrm{G}$ frequencies that will be assigned in Colombia; however, MinICT advances studies of viability about spectrum portions that could serve as IMT (International Mobile Telecommunications) bands, which should finalize in the fourth trimester of 2021 [28]. Also, in June 2020, MinICT invited the operators to manifest their interest to participate in processes of permission, obtaining radioelectric spectrum use for the provision of IMT services in the $700 \mathrm{MHZ}, 1900 \mathrm{MHZ}, 2500 \mathrm{MHz}$, and $3500 \mathrm{MHz}$ bands [29]. 
Table 3. General description of characteristics/typical requirements of IoT applications. Own elaboration based on information from MinICT and the National Spectrum Agency (ANE) and [17].

\begin{tabular}{|c|c|c|c|c|}
\hline Band Type & Bands & Current Usage & Advantages & Disadvantages \\
\hline Less than $1 \mathrm{GHz}$ & $\begin{array}{l}614-698 \mathrm{MHz} \\
698-806 \mathrm{MHz}\end{array}$ & $\begin{array}{l}\text { Broadcasting and } \\
\text { mobile service } \\
\text { Mobile service }\end{array}$ & $\begin{array}{c}\text { Greater coverage than } \\
\text { frequencies above } \\
1 \mathrm{GHz}\end{array}$ & $\begin{array}{c}\text { Lower channel capacity } \\
\text { (bps) than frequencies } \\
\text { above } 1 \mathrm{GHz}\end{array}$ \\
\hline $\begin{array}{l}\text { Between } 1 \text { and } \\
\quad 6 \mathrm{GHz}\end{array}$ & 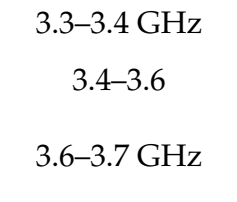 & $\begin{array}{l}\text { Mobile service } \\
\text { Fixed service, mobile service, } \\
\text { and satellite service } \\
\text { Fixed service, mobile service, } \\
\text { and satellite service }\end{array}$ & $\begin{array}{l}\text { Balance between } \\
\text { coverage and capacity }\end{array}$ & $\begin{array}{c}\text { Saturated spectrum for } \\
\text { mobile and } \\
\text { wireless services }\end{array}$ \\
\hline Over $6 \mathrm{GHz}$ & $\begin{array}{c}24.25-27.5 \mathrm{GHz} \\
26.5-29.5 \mathrm{GHz} \\
31.8-33.4 \mathrm{GHz} \\
37-40.5 \mathrm{GHz} \\
40.5-42.5 \mathrm{GHz} \\
42.5-43.5 \mathrm{GHz} \\
45.5-47 \mathrm{GHz} \\
47-47.2 \mathrm{GHz} \\
47.2-50.2 \mathrm{GHz} \\
50.4-52.6 \mathrm{GHz} \\
66-71 \mathrm{GHz} \\
71-76 \mathrm{GHz} \\
81-86 \mathrm{GHz}\end{array}$ & $\begin{array}{c}\text { Fixed service, mobile service, } \\
\text { and radio navigation } \\
\text { Fixed service, mobile service, } \\
\text { and satellite service } \\
\text { Fixed service, radio navigation, } \\
\text { and space research } \\
\text { Fixed service, mobile service, } \\
\text { and satellite service } \\
\text { Fixed service, mobile service, } \\
\text { satellite service, } \\
\text { and broadcasting } \\
\text { Fixed service, mobile service, } \\
\text { satellite service, and } \\
\text { radio astronomy } \\
\text { Mobile service, satellite service, } \\
\text { and radio navigation } \\
\text { Amateur } \\
\text { Fixed service, mobile service, } \\
\text { and satellite service } \\
\text { Fixed service, mobile service, } \\
\text { and satellite service } \\
\text { Fixed service, mobile service, } \\
\text { satellite service, and } \\
\text { radio navigation } \\
\text { Fixed service, mobile service, } \\
\text { satellite service, } \\
\text { and broadcasting } \\
\text { Fixed service, mobile service, } \\
\text { satellite service, and } \\
\text { radio astronomy }\end{array}$ & $\begin{array}{l}\text { Channel availability } \\
\text { increased and spectrum } \\
\text { saturation reduced }\end{array}$ & $\begin{array}{c}\text { Lower coverage than } \\
\text { other bands } \\
\text { aforementioned }\end{array}$ \\
\hline
\end{tabular}

Other bands that are considered by MinICT in the $5 \mathrm{G}$ Plan $(31 \mathrm{GHz}, 40 \mathrm{GHz}, 71 \mathrm{GHz}$, and $81 \mathrm{GHz}$ ) were not identified by the WCR-19 for the provided 5G service. However, they could be a part of future spectrum auctions thanks to the developments of manufacturers and the needs of operators. Between the examples of this situation, there is the band of $28 \mathrm{GHz}$, not identified by the WCR-19 inside the set of destined frequencies to IMT, in which the $5 \mathrm{G}$ is framed. However, as it can be observed in Table 2, some countries have already auctioned in this spectrum portion, and others have manifested interest on doing so in the future.

\subsection{Projected 5G Frequency Bands for Use in Colombia}

In April 2020, MinICT authorized 5G pilot tests in Colombia in five (05) bands of the spectrum: 3.5-3.7 GHz, 24.25-27.5 GHz, 37-43.5 GHz, 45.5-47 GHz, and 47.2-48.2 GHz. Nevertheless, in agreement with the inform of assignation of the direction of the industry of Communications of MinICT, four of the network providers and telecommunication systems (NPTS) operating in Colombia submitted applications to perform tests in the band of 
$3500 \mathrm{MHz}$ to $3600 \mathrm{MHz}$, while others with an interest submitted their application in bands from $3300 \mathrm{MHz}$ to $3400 \mathrm{MHz}$ and from 587 to $592 \mathrm{MHz}$ [30]. In total, 52 entities (public and private) and 24 natural persons showed an interest in participating in this process for use in eight different types of activities in the next descending order of demand [17]: The first is the interest in using it in projects related to smart cities, followed by its use in applications in education, agricultural development, the entertainment industry, virtual reality, public safety, the health sector, and finally, in transportation.

Of the operators with participation in the mobile services in Colombia, three of them submitted applications to carry out pilot tests: Colombia Telecommunications, COMCEL Cellular Communication (Claro), and the Bogota Telecommunications Company. In those tests, the Claro company recorded speeds from $864 \mathrm{Mbps}$ in the download link (DL) and $103 \mathrm{Mbps}$ in the upload link (UL) [31]. The Movistar company, in alliance with the military hospital and with the MinICT endorsement, incorporated this technology to a telemedicine car with the purpose of providing services of interconsultations and an observation of procedures through video conferences. The results show that they reached rates of $1.62 \mathrm{Gbps}$ in DL and $176 \mathrm{Mbps}$ in UL [32]. Other values obtained by mobile telephone providers in Colombia, before the permissions granted in 2020 in indoor scenarios, reached speeds of $640 \mathrm{Mbps}$ per cell and a spectral efficiency of $32 \mathrm{Mbps}$ per MHz [33]. The Claro company in 2018 had speeds of $10 \mathrm{Gbps}$ in the $28 \mathrm{GHz}$, and a latency lower than $1 \mathrm{~ms}$ [34].

Regarding the auctions of spectrum destined for $5 \mathrm{G}$ in Colombia, there is no set date, although there are some projections. The ANE has suggested that, with the entry of 5G, a spectral portion of $400 \mathrm{MHz}$ is necessary in the $\mathrm{C}$ band (3300-3700 MHz) in 2018, and close to $1172 \mathrm{MHz}$ in the year 2024 [35]. Figure 2a,b shows a projection of ANE for the demand for mobile technology of its bandwidths in MHz until the year 2029, and the expectations for future spectrum auctions in the country.

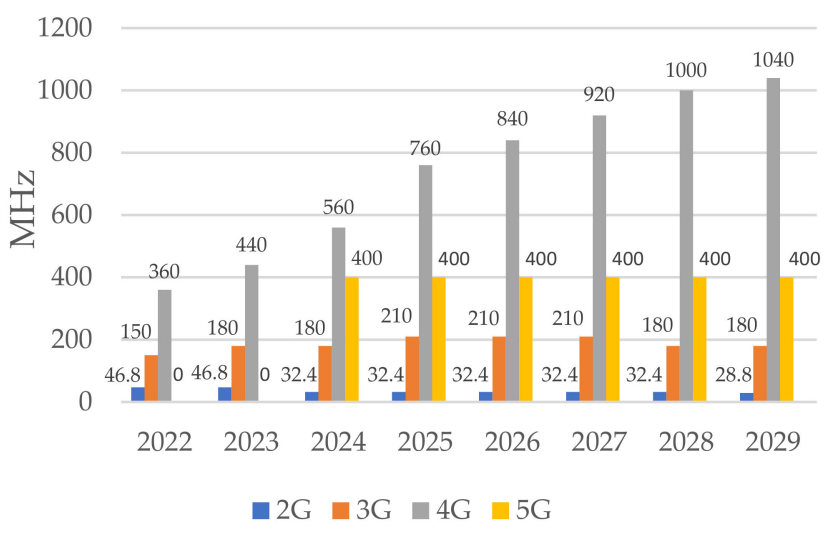

Year

(a)

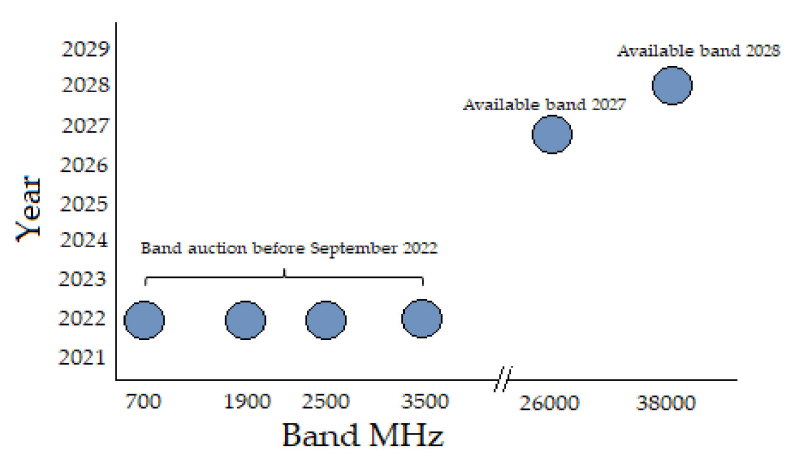

(b)

Figure 2. (a) Prospective spectrum demand for Colombia IMT, and (b) Band auctions projections. Source: ANE and [17].

Two scenarios have been put forward for 5G implementation from 4G-LTE: StandAlone (SA) and Non-StandAlone (NSA) [17]. Regarding SA, these are radio access architectures that do not use the existing $4 \mathrm{G}$ networks core, while in NSA, the $5 \mathrm{G}$ architecture is based on a $4 \mathrm{G}$ network core. In the Colombian case, it is expected that the first commercial networks will start in NSA mode, and that is why MinICT has been strengthening the $4 \mathrm{G}$ expansion process in Colombia, for which spectrum auction processes have already been carried out in the $700 \mathrm{MHz}$ and $2500 \mathrm{MHz}$ bands to expand mobile broadband coverage to 3658 locations located in rural areas. In addition, operators Tigo and Claro will execute the 
transition from 2G and 3G to 4G within four years, starting in 2021 [36,37]. Besides, in 2020, Colombia opened a consultation for expressions of interest in obtaining radio spectrum for the provision of IMT, of which $5 \mathrm{G}$ is a part. The bands destined for a future granting of permits are: $700 \mathrm{MHz}, 1900 \mathrm{MHz}, 2500 \mathrm{MHz}$, and $3500 \mathrm{MHz}$ [29].

IoT is largely responsible for this increase in spectrum requirements in $5 \mathrm{G}$, and this is demonstrated by the forecasts of different entities [38-40]. For example, CISCO, in its annual internet report, predicts that in the year 2023, the number of M2M (machine-tomachine) connections will be 14.7 billion, with an average of 1.8 connections per user worldwide [41]. In this report, they also present figures about the IoT applications with the highest number of connections, these being those related to the home. In addition, smart city and vehicle related applications will be faster growing.

Regarding IoT in Colombia, in accordance with the 5G Plan and in harmony with world trends, MinICT proposes possible uses in each of the 5G spectrum portions. Frequencies below $1 \mathrm{GHz}$ will preferably be used for high-speed mobile broadband in urban, suburban, and rural areas, in order to serve the deployment of IoT services. Regarding the band between 1 and $6 \mathrm{GHz}$, it is the one with the greatest options to be used in the first commercial 5G implementations, and has $3 \mathrm{G}$ and $4 \mathrm{G}$ service assignments. As for the frequency band above $6 \mathrm{GHz}$ (with or without a license), its use is proposed for ultra-fast speed connections.

A study presented by the General Directorate of Communications Networks, Contents, and Technology (an official entity of the European Community) indicated that approximately $19 \mathrm{GHz}$ of shared spectrum could be required for IoT in the coming years [42]. The same report shows that there is not enough spectrum in the lower $1 \mathrm{GHz}$ bands (low band) and between 1 to $6 \mathrm{GHz}$ (mid-band) if you want to develop monitoring applications for highways, power supplies, and healthcare. It is important to highlight that they arrived at this result from three factors: (a) total number of devices per $\mathrm{km} 2$, (b) data rate of the devices, and (c) spectral efficiency. In terms of spectrum usage preferences in IoT, mobile operators prefer to implement IoT within licensed spectrum, while manufacturers lean towards implementation in unlicensed bands to avoid licensing costs [43].

\subsection{Opportunities for Using IoT/5G Applications for Projects in Colombia}

The National Development Plan (NDP) is an official document prepared by the National Planning Department of Colombia, which contains the strategic guidelines of each government policy [44]. It is a roadmap that details various aspects for improvement, projected for a period of four years. It is issued at the beginning of the mandate of each presidential government in order to ensure the continuous progress of the nation in different aspects, such as social, economic, environmental, and health. In this sense, one of the goals in the 2018-2022 four-year period is the increase in the number of internet connections and the digital transformation of society [45]. Our research is focused on the areas where the explosive growth of IoT applications is envisaged to work in conjunction with 5G wireless networks to become ideal solutions in different fields of national development.

Administratively, Colombia is made up of departments, districts, and municipalities in its political division with the largest geographical extension. However, historically it has also been subdivided into six (06) natural regions: Amazon, Andean, Caribbean, Island, Orinoco, and Pacific, which can be seen in Figure 3. Each of them is made up of different departments with their own geographical, social, and cultural characteristics that differentiate them from each other. Although there is an NDP, each department draws up its own government policy through a Departmental Development Plan (DDP), which is a planning instrument for a four-year period that allows governors to set the objectives and goals of their period, starting from its potentialities and shortcomings [46]. Through the DDPs, the aim is to strengthen different economic activities that help improve the quality of life of its citizens, and within this framework, IoT and 5G are promising tools that could help generate innovation and solutions to different problems. This section presents a summary of the economic activities in which each department has proposed its development efforts 
in the 2020-2023 period (see Table 4), and subsequently, the potentialities of IoT framed in $5 \mathrm{G}$ are analyzed for the advancement of the regions.

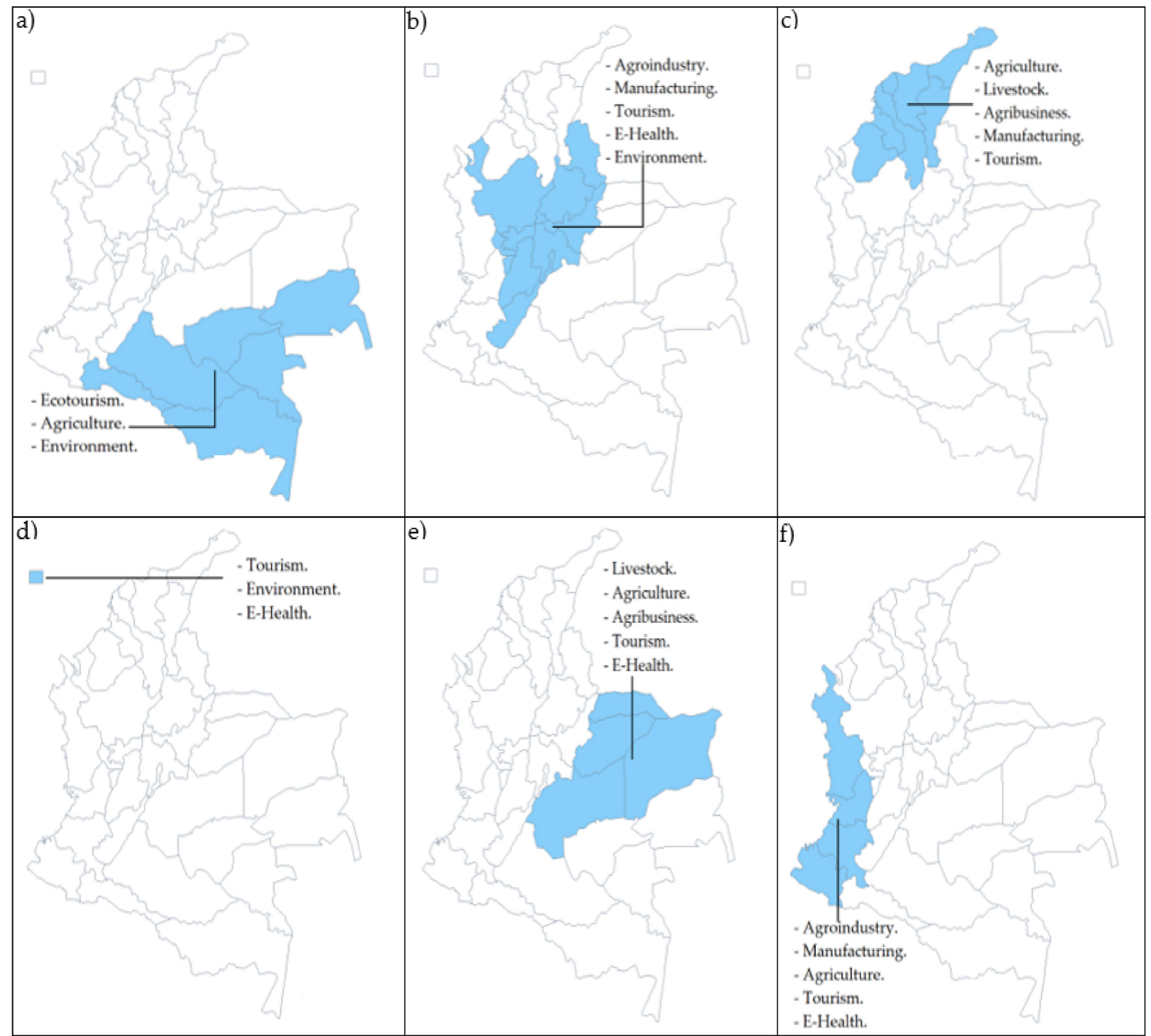

Figure 3. Potential demand for IoT with 5G in natural regions of Colombia according to DDPs: (a) Amazon, (b) Andean, (c) Caribbean, (d) Island, (e) Orinoco, and (f) Pacific. 
Table 4. Economic activities, problematics, and potentialities of Colombia departments.

\begin{tabular}{|c|c|c|c|c|c|c|c|}
\hline \multicolumn{8}{|c|}{ The Caribbean Region } \\
\hline Department & Agriculture & Tourism & Cattle Raising & Health & Industry & Environmental & References \\
\hline Atlántico & $\begin{array}{l}\text { Corn, yucca, mango, } \\
\text { citrus, pigeon pea, } \\
\text { sorghum, melon }\end{array}$ & $\begin{array}{l}\text { Health tourism, Barranquilla } \\
\text { Carnival, Barranquilla Zoo, } \\
\text { Santa Verónica beaches }\end{array}$ & $\begin{array}{l}\text { Fish farming, poultry } \\
\text { farming, cattle, } \\
\text { pigs, sheep }\end{array}$ & $\begin{array}{c}\text { Mortality from } \\
\text { malignant tumors, } \\
\text { mortality from ischemic } \\
\text { heart diseases }\end{array}$ & $\begin{array}{l}\text { Manufacturing, cement, } \\
\text { agribusiness, } \\
\text { chemical, port, } \\
\text { electricity generation }\end{array}$ & $\begin{array}{l}\text { Erosion, contamination } \\
\text { of water sources, floods, } \\
\text { forest fires }\end{array}$ & {$[47-57]$} \\
\hline Guajira & $\begin{array}{l}\text { Corn, coffee, yucca, rice, } \\
\text { banana, bean, oil palm }\end{array}$ & Natural parks & $\begin{array}{l}\text { Aquaculture, goats, } \\
\text { sheep, equine cattle, } \\
\text { fish farming }\end{array}$ & $\begin{array}{c}\text { Communicable diseases, } \\
\text { diseases of the } \\
\text { circulatory system }\end{array}$ & $\begin{array}{l}\text { Mining, port electricity } \\
\text { generation, salinera }\end{array}$ & Desertification & {$[50,54-56,58-61]$} \\
\hline Cesar & $\begin{array}{l}\text { Palm oil, yucca, banana, } \\
\text { rice, corn, pin, melon }\end{array}$ & $\begin{array}{c}\text { Vallenato festival, ecotourism } \\
\text { and ethno-tourism in the Sierra } \\
\text { Nevada de Santa Marta and } \\
\text { Serranía del Perijá, Ciénaga De } \\
\text { la Zapatosa }\end{array}$ & $\begin{array}{l}\text { Sheep, goat, cattle, fish } \\
\text { farming, poultry }\end{array}$ & $\begin{array}{l}\text { Maternal mortality, } \\
\text { infant mortality from } \\
\text { Acute respiratory } \\
\text { infection (ARI), infant } \\
\text { mortality from acute } \\
\text { diarrheal disease (ADD) }\end{array}$ & Mining, agribusiness & $\begin{array}{l}\text { Impact by climate } \\
\text { change, impact by } \\
\text { natural disasters, } \\
\text { deforestation }\end{array}$ & {$[50,52-54,66-68]$} \\
\hline Córdoba & $\begin{array}{l}\text { Corn, banana, yucca, } \\
\text { rice, yam, cotton, oil } \\
\text { palm, cocoa }\end{array}$ & $\begin{array}{l}\text { Ciénagas, Montería cattle fair, } \\
\text { joint festival in San Pelayo } \\
\text { natural parks, Gulf of } \\
\text { Morrosquillo beaches }\end{array}$ & $\begin{array}{l}\text { Cattle, pigs, equine } \\
\text { cattle, sheep, goats, } \\
\text { poultry, aquaculture, } \\
\text { fish farming, } \\
\text { beekeeping }\end{array}$ & $\begin{array}{l}\text { High operational costs } \\
\text { due to geographic } \\
\text { conditions, ADD, } \\
\text { obesity, diabetes, high } \\
\text { blood pressure }\end{array}$ & $\begin{array}{l}\text { Agribusiness, mining, } \\
\text { electricity generation }\end{array}$ & $\begin{array}{l}\text { Air pollution from } \\
\text { mining exploitation, } \\
\text { GHG emissions, } \\
\text { deforestation, illegal } \\
\text { hunting }\end{array}$ & {$[50-55,61,65,69,70]$} \\
\hline Magdalena & $\begin{array}{l}\text { Palm oil, corn, yucca, } \\
\text { banana, coffee, citrus, } \\
\text { mango, plantain }\end{array}$ & $\begin{array}{l}\text { Hotels, Tayrona Park, Sierra } \\
\text { Nevada de Santa Marta } \\
\text { National Natural Park, Santa } \\
\text { Marta and El Rodadero } \\
\text { beaches, ecotourism }\end{array}$ & $\begin{array}{l}\text { Marine fishing, cattle, } \\
\text { sheep, poultry, } \\
\text { fish farming }\end{array}$ & $\begin{array}{c}\text { ARI, ADD, } \\
\text { genitourinary diseases, } \\
\text { cardiovascular diseases }\end{array}$ & $\begin{array}{l}\text { Manufacturing, port } \\
\text { industry }\end{array}$ & $\begin{array}{l}\text { Deforestation, } \\
\text { inappropriate land use }\end{array}$ & {$[50,52-54,60,71-73]$} \\
\hline Sucre & $\begin{array}{l}\text { Rice, yucca, corn, yam, } \\
\text { banana, oil palm, pin }\end{array}$ & $\begin{array}{l}\text { Gulf of Morrosquillo Beaches, } \\
\text { January } 20 \text { festivities in } \\
\text { Sincelejo, San Bernardo } \\
\text { Islands Archipelago }\end{array}$ & $\begin{array}{l}\text { Cattle, equine cattle, } \\
\text { pigs, poultry, fish } \\
\text { farming, beekeeping }\end{array}$ & ADD, ARI, dengue & $\begin{array}{l}\text { Agroindustry, mining, } \\
\text { cement industry, } \\
\text { port industry }\end{array}$ & $\begin{array}{l}\text { Illegal hunting, over } \\
\text { exploitation of water } \\
\text { sources, forest fires, } \\
\text { deforestation, soil } \\
\text { deterioration, } \\
\text { pesticide contamination }\end{array}$ & {$[50,51,56,61,65,74,75]$} \\
\hline
\end{tabular}


Table 4. Cont.

\begin{tabular}{|c|c|c|c|c|c|c|c|}
\hline \multicolumn{8}{|c|}{ Andean Region } \\
\hline Department & Agriculture & Tourism & Cattle raising & Health & Industry & Environmental & References \\
\hline Antioquia & $\begin{array}{l}\text { Coffee, banana, cane, } \\
\text { plantain, cocoa, corn, } \\
\text { rice, flowers }\end{array}$ & $\begin{array}{c}\text { Flower Festival, hotels, } \\
\text { ecotourism, cultural tourism, } \\
\text { health tourism }\end{array}$ & $\begin{array}{l}\text { Swine, cattle, equine } \\
\text { cattle, sheep, goats, } \\
\text { poultry, fish farming, } \\
\text { beekeeping }\end{array}$ & $\begin{array}{l}\text { Cardiovascular diseases, } \\
\text { respiratory diseases, } \\
\text { hypertension, } \\
\text { infrastructure problems }\end{array}$ & $\begin{array}{l}\text { Manufacturing industry, } \\
\text { agribusiness, mining, } \\
\text { Center for the Fourth } \\
\text { Industrial Revolution, } \\
\text { electricity generation, } \\
\text { port industry }\end{array}$ & $\begin{array}{l}\text { GHG emissions, climate } \\
\text { change risks, } \\
\text { deforestation, illegal } \\
\text { hunting }\end{array}$ & $\begin{array}{c}{[50-52,54,55,57,61,65,76-} \\
78]\end{array}$ \\
\hline Boyacá & $\begin{array}{c}\text { Potato, vegetables, } \\
\text { cocoa, fruit trees, } \\
\text { panelera cane, quinoa, } \\
\text { cereals }\end{array}$ & $\begin{array}{l}\text { Villa de Leyva, Chicamocha } \\
\text { Canyon, Tunja, El Cocuy } \\
\text { National Natural Park, Tota } \\
\text { Lake. }\end{array}$ & $\begin{array}{l}\text { Cattle, sheep-goat, } \\
\text { beekeeping, poultry, pig } \\
\text { and fish farming. }\end{array}$ & $\begin{array}{l}\text { Diseases of the } \\
\text { circulatory system, } \\
\text { neoplasms }\end{array}$ & $\begin{array}{l}\text { Agribusiness, mining, } \\
\text { manufacturing industry, } \\
\text { electricity generation }\end{array}$ & $\begin{array}{l}\text { Deforestation, risks due } \\
\text { to climate change }\end{array}$ & {$[50,52-54,57,65,79,80]$} \\
\hline Caldas & $\begin{array}{l}\text { Coffee, banana, } \\
\text { panelera cane, avocado, } \\
\text { citrus, cocoa, sugarcane }\end{array}$ & $\begin{array}{l}\text { Coffee cultural landscape, } \\
\text { avitourism, nature tourism, hot } \\
\text { springs }\end{array}$ & $\begin{array}{l}\text { Cattle, swine, fish } \\
\text { farming, poultry } \\
\text { farming, }\end{array}$ & $\begin{array}{l}\text { Diseases of the } \\
\text { circulatory system, } \\
\text { diabetes mellitus }\end{array}$ & $\begin{array}{c}\text { Agribusiness, } \\
\text { metalworking industry, } \\
\text { manufacturing industry, } \\
\text { textile industry, } \\
\text { electricity generation }\end{array}$ & $\begin{array}{c}\text { Soil loss due to various } \\
\text { anthropic activities, } \\
\text { water bodies affected by } \\
\text { discharges. } \\
\text { deforestation }\end{array}$ & {$[50-53,55,57,81,82]$} \\
\hline Cundinamarca & $\begin{array}{l}\text { Potato, carrot, tomato, } \\
\text { onion, lettuce, Corn, } \\
\text { banana, sugar cane, } \\
\text { flowers }\end{array}$ & $\begin{array}{l}\text { Hotels, ecotourism, natural } \\
\text { parks, forest reserves. }\end{array}$ & $\begin{array}{l}\text { Cattle farming, sheep } \\
\text { farming, goat farming, } \\
\text { equine farming, pig } \\
\text { farming, poultry } \\
\text { farming, fish farming, } \\
\text { beekeeping }\end{array}$ & $\begin{array}{l}\text { Lack of insurance and } \\
\text { access to health services } \\
\text { in the municipalities }\end{array}$ & $\begin{array}{l}\text { Manufacturing industry, } \\
\text { agribusiness, chemical } \\
\text { industry, electricity } \\
\text { generation }\end{array}$ & $\begin{array}{l}\text { Degradation of water } \\
\text { reserve areas, risks due } \\
\text { to climate change, forest } \\
\text { fires, contamination of } \\
\text { water sources }\end{array}$ & {$[50-55,57,61,65,77,83]$} \\
\hline Huila & $\begin{array}{l}\text { coffee, rice, banana, } \\
\text { beans, corn, sugar cane, } \\
\text { cocoa, yucca }\end{array}$ & $\begin{array}{l}\text { Archaeological tourism in San } \\
\text { Agustín, nature tourism in the } \\
\text { Tatacoa desert, ecotourism }\end{array}$ & $\begin{array}{l}\text { Cattle, swine, fish } \\
\text { farming, poultry } \\
\text { farming, beekeeping }\end{array}$ & $\begin{array}{l}\text { Diseases of the } \\
\text { circulatory system, } \\
\text { neoplasms, ARI in } \\
\text { children under } 5 \text { years } \\
\text { of age, ADD }\end{array}$ & $\begin{array}{l}\text { Agribusiness, mining, } \\
\text { electricity generation }\end{array}$ & $\begin{array}{l}\text { Impacts of climate } \\
\text { change, contamination } \\
\text { of water sources, loss of } \\
\text { strategic ecosystems }\end{array}$ & {$[50-53,55,65,84,85]$} \\
\hline $\begin{array}{l}\text { Norte de } \\
\text { Santander }\end{array}$ & $\begin{array}{l}\text { Coffee, cocoa, oil palm, } \\
\text { sugar cane, banana, } \\
\text { avocado, rice, beans }\end{array}$ & Ecotourism, Villa del Rosario & $\begin{array}{l}\text { Cattle, equine cattle, } \\
\text { pigs, sheep, goats, } \\
\text { poultry }\end{array}$ & $\begin{array}{l}\text { Access to health in rural } \\
\text { areas is limited, leprosy, } \\
\text { malaria, dengue }\end{array}$ & $\begin{array}{c}\text { Agribusiness, } \\
\text { manufacturing industry, } \\
\text { electricity generation }\end{array}$ & $\begin{array}{l}\text { Pollution of water } \\
\text { sources, deforestation }\end{array}$ & {$[50,51,53-55,61,86,87]$} \\
\hline Quindío & $\begin{array}{l}\text { Banana, coffee, citrus, } \\
\text { avocado, banana }\end{array}$ & $\begin{array}{c}\text { Parque del Café, coffee cultural } \\
\text { landscape, ecotourism }\end{array}$ & Poultry, swine, cattle & $\begin{array}{l}\text { They need to strengthen } \\
\text { the hospital network }\end{array}$ & Agroindustry & Deforestation & {$[50-52,88-90]$} \\
\hline Risaralda & $\begin{array}{c}\text { Coffee, banana, } \\
\text { avocado, sugarcane, } \\
\text { corn, beans, tomato, } \\
\text { onion and various } \\
\text { vegetables }\end{array}$ & $\begin{array}{l}\text { Coffee cultural landscape, } \\
\text { natural parks, ecotourism, hot } \\
\text { springs of Santa Rosa and San } \\
\text { Vicente }\end{array}$ & $\begin{array}{l}\text { Cattle, pig farming, fish } \\
\text { farming, poultry } \\
\text { farming }\end{array}$ & $\begin{array}{l}\text { Diseases of the } \\
\text { circulatory system, } \\
\text { neoplasms, diseases of } \\
\text { the respiratory system }\end{array}$ & $\begin{array}{c}\text { Agribusiness, } \\
\text { manufacturing industry, }\end{array}$ & $\begin{array}{c}\text { Deforestation, } \\
\text { contamination of water } \\
\text { sources, loss of soil, air } \\
\text { pollution, illegal } \\
\text { hunting }\end{array}$ & {$[51-53,57,91,92]$} \\
\hline
\end{tabular}


Table 4. Cont.

\begin{tabular}{|c|c|c|c|c|c|c|c|}
\hline \multicolumn{8}{|c|}{ The Andean Region } \\
\hline Department & Agriculture & Tourism & Cattle Raising & Health & Industry & Environmental & References \\
\hline Santander & $\begin{array}{c}\text { Palm oil, cocoa, coffee, } \\
\text { sugarcane, citrus, } \\
\text { banana, rubber, } \\
\text { pineapple, Yucca }\end{array}$ & $\begin{array}{l}\text { Barichara, San Gil, Chicamocha } \\
\text { National Park, natural parks, } \\
\text { ecotourism, health tourism }\end{array}$ & $\begin{array}{l}\text { Cattle, pig, equine, goat, } \\
\text { sheep, poultry, fish } \\
\text { farming }\end{array}$ & $\begin{array}{c}\text { Heart disease and } \\
\text { neoplasms are common } \\
\text { causes of death. Public } \\
\text { health strategies that } \\
\text { stimulate sport will be } \\
\text { worked on }\end{array}$ & $\begin{array}{c}\text { Mining, oil, } \\
\text { petrochemical, } \\
\text { agribusiness, } \\
\text { manufacturing, } \\
\text { electricity generation, } \\
\text { port }\end{array}$ & $\begin{array}{c}\text { Deforestation, } \\
\text { contamination of water } \\
\text { sources }\end{array}$ & {$[50-57,61,93-95]$} \\
\hline Tolima & $\begin{array}{l}\text { Coffee, rice, corn, } \\
\text { banana, beans, sugar } \\
\text { cane, avocado, cocoa, } \\
\text { sugar cane, mango }\end{array}$ & $\begin{array}{l}\text { Ecotourism, adventure tourism } \\
\text { and historical tourism }\end{array}$ & $\begin{array}{l}\text { Bovine, equine, ovine, } \\
\text { pig and poultry } \\
\text { farming, fish farming }\end{array}$ & $\begin{array}{l}\text { ARI, ADD, syphilis, } \\
\text { diseases of the circulatory } \\
\text { system }\end{array}$ & $\begin{array}{c}\text { Manufacturing } \\
\text { industries, agribusiness, } \\
\text { mining, electricity } \\
\text { generation }\end{array}$ & $\begin{array}{l}\text { Risks due to climate } \\
\text { change, contamination } \\
\text { of water sources, } \\
\text { deforestation }\end{array}$ & {$[50-55,61,96-98]$} \\
\hline \multicolumn{8}{|c|}{ Amazon Region } \\
\hline Department & Agriculture & Tourism & Cattle raising & Health & Industry & Environmental & References \\
\hline Amazonas & $\begin{array}{l}\text { Yucca, banana, various } \\
\text { fruit trees }\end{array}$ & $\begin{array}{l}\text { Flor de Loto Nature Reserve, } \\
\text { Los Micos Island, indigenous } \\
\text { communities, Lagos de Tarapoto }\end{array}$ & $\begin{array}{l}\text { Swine, poultry, fish } \\
\text { farming }\end{array}$ & $\begin{array}{l}\text { Leptospirosis, diabetes, high } \\
\text { blood pressure }\end{array}$ & manufacture & $\begin{array}{l}\text { Deforestation, risks due } \\
\text { to climate change }\end{array}$ & {$[52,99,100]$} \\
\hline Caquetá & $\begin{array}{l}\text { Banana, yucca, cocoa, } \\
\text { rubber, coffee, cane, rice, } \\
\text { corn }\end{array}$ & $\begin{array}{c}\text { Ecotourism, adventure tourism, } \\
\text { ethno-tourism }\end{array}$ & $\begin{array}{l}\text { Cattle, swine, poultry, } \\
\text { sheep }\end{array}$ & $\begin{array}{l}\text { Lack of access to services, } \\
\text { cardiovascular diseases, ARI }\end{array}$ & Agroindustry & $\begin{array}{l}\text { Low quality of water for } \\
\text { human consumption, } \\
\text { contamination by } \\
\text { chemical substances }\end{array}$ & {$[50,51,53,54,101]$} \\
\hline Guainía & $\begin{array}{l}\text { Banana, yucca, corn, } \\
\text { cocoa }\end{array}$ & $\begin{array}{l}\text { Ecotourism, Puinawual Natural } \\
\text { Reserve, Cerros de Manicure }\end{array}$ & Swine, poultry, fishing & $\begin{array}{l}\text { Tuberculosis, ARI, ischemic } \\
\text { heart disease }\end{array}$ & Mining & $\begin{array}{l}\text { Deforestation, effects of } \\
\text { climate change }\end{array}$ & {$[51,52,102]$} \\
\hline Guaviare & $\begin{array}{l}\text { Corn, banana, yucca, } \\
\text { rice, rubber, cane, cocoa }\end{array}$ & $\begin{array}{l}\text { Ecotourism, Serranía de } \\
\text { Chiribiquete Natural Park }\end{array}$ & $\begin{array}{l}\text { Cattle, equine cattle, } \\
\text { poultry }\end{array}$ & $\begin{array}{c}\text { Lack of access to services, } \\
\text { diabetes, }\end{array}$ & $\begin{array}{l}\text { Mining, manufacturing } \\
\text { industries }\end{array}$ & Deforestation & {$[52,61,103-105]$} \\
\hline Putumayo & $\begin{array}{l}\text { Yucca, corn, cacao, cane, } \\
\text { chontaduro, pepper, } \\
\text { cacao, banana }\end{array}$ & Ecotourism & $\begin{array}{l}\text { Poultry, cattle, equine } \\
\text { cattle, pig farming, fish } \\
\text { farming }\end{array}$ & $\begin{array}{l}\text { Diseases of the circulatory } \\
\text { system, ARI, HIV, intestinal } \\
\text { infectious diseases }\end{array}$ & Mining, agribusiness & Deforestation, floods & {$[50-53,61,106,107]$} \\
\hline Vaupés & Yucca, cocoa & $\begin{array}{l}\text { Ecotourism, ethno-tourism, } \\
\text { nature reserves }\end{array}$ & Aquaculture & $\begin{array}{l}\text { Lack of access to services, } \\
\text { diseases of the circulatory } \\
\text { system }\end{array}$ & Mining & $\begin{array}{l}\text { Deforestation, forest } \\
\text { fires }\end{array}$ & [108] \\
\hline \multicolumn{8}{|c|}{ Island Region } \\
\hline Department & Agriculture & Tourism & Cattle raising & Health & Industry & Environmental & References \\
\hline \multirow[t]{2}{*}{$\begin{array}{l}\text { San Andrés, } \\
\text { Providencia y } \\
\text { Santa Catalina }\end{array}$} & $\begin{array}{l}\text { Coconut, yam, banana, } \\
\text { yucca, corn }\end{array}$ & Hotels, beaches, ecotourism & Fishing, pigs, poultry & $\begin{array}{l}\text { Obsolescence of } \\
\text { infrastructure, diseases of } \\
\text { the }\end{array}$ & Manufacturing, port & $\begin{array}{l}\text { Effects of climate } \\
\text { change, pollution of } \\
\text { water sources, }\end{array}$ & {$[52,56,109]$} \\
\hline & & & & $\begin{array}{l}\text { circulatory system, } \\
\text { neoplasms }\end{array}$ & & $\begin{array}{l}\text { pollution of marine } \\
\text { ecosystems }\end{array}$ & \\
\hline
\end{tabular}


Table 4. Cont.

\begin{tabular}{|c|c|c|c|c|c|c|c|}
\hline \multicolumn{8}{|c|}{ Pacific Region } \\
\hline Department & Agriculture & Tourism & Cattle raising & Health & Industry & Environmental & References \\
\hline Cauca & $\begin{array}{l}\text { Sugar cane, coffee, } \\
\text { banana, sugar cane, } \\
\text { corn, yucca }\end{array}$ & Holy week Popayán & $\begin{array}{l}\text { Cattle, equine cattle, } \\
\text { sheep, pig farming, } \\
\text { poultry farming, fish } \\
\text { farming, beekeeping }\end{array}$ & ARI, ADD & $\begin{array}{c}\text { Agribusiness, } \\
\text { manufacturing, } \\
\text { electricity generation }\end{array}$ & Deforestation. & $\frac{[51-}{55,57,61,63,110,111]}$ \\
\hline Chocó & $\begin{array}{l}\text { Banana, corn, rice, } \\
\text { cocoa and coconut. }\end{array}$ & $\begin{array}{l}\text { Virgin beaches, ecotourism, } \\
\text { adventure tourism. }\end{array}$ & $\begin{array}{l}\text { Cattle, poultry farming, } \\
\text { fish farming. }\end{array}$ & Lack of access to services. & Gold mining & $\begin{array}{c}\text { Deforestation and water } \\
\text { quality. }\end{array}$ & {$[50,52,53,112]$} \\
\hline Nariño & $\begin{array}{l}\text { Coffee, potato, banana, } \\
\text { cocoa, oil palm, sugar } \\
\text { cane, pea, corn, coconut, } \\
\text { beans }\end{array}$ & $\begin{array}{c}\text { Black and white carnival, Our } \\
\text { Lady of Las Lajas Sanctuary, } \\
\text { Doña Juana Volcanic Complex } \\
\text { Natural Park }\end{array}$ & $\begin{array}{l}\text { Cattle, equine cattle, } \\
\text { sheep, marine fishing, } \\
\text { fish farming, pig } \\
\text { farming, poultry } \\
\text { farming }\end{array}$ & Child mortality & Mining, port & Deforestation & [50-55,61,113-115] \\
\hline Valle del Cauca & $\begin{array}{l}\text { Sugar cane, coffee, } \\
\text { banana, corn, sugar } \\
\text { cane, citrus, plantain, } \\
\text { rice, pineapple, avocado }\end{array}$ & Cali Fair, health tourism. & $\begin{array}{l}\text { Cattle, poultry, swine, } \\
\text { fish farming, } \\
\text { beekeeping }\end{array}$ & Infant mortality, violence. & $\begin{array}{c}\text { Agribusiness, } \\
\text { manufacturing, rubber, } \\
\text { port, chemical, } \\
\text { electricity generation }\end{array}$ & Deforestation & $\begin{array}{c}{[50-53,55-} \\
57,65,116,117]\end{array}$ \\
\hline \multicolumn{8}{|c|}{ Orinoco Region } \\
\hline Department & Agriculture & Tourism & Cattle raising & Health & Industry & Environmental & References \\
\hline Casanare & $\begin{array}{l}\text { Rice, oil palm, coffee, } \\
\text { corn, banana, Yucca }\end{array}$ & Eastern plains & $\begin{array}{l}\text { Cattle, equine cattle, } \\
\text { sheep, pig farming, } \\
\text { poultry farming, fish } \\
\text { farming }\end{array}$ & $\begin{array}{l}\text { Deficiency in access to } \\
\text { health services. }\end{array}$ & Agribusiness, mining & Deforestation. & {$[50-54,61,120,121]$} \\
\hline Meta & $\begin{array}{l}\text { Palm oil, corn, sugar } \\
\text { cane, rice, soy, banana. }\end{array}$ & Natural parks, caño crystals. & $\begin{array}{l}\text { Fish farming, } \\
\text { beekeeping, cattle, } \\
\text { sheep, goats }\end{array}$ & $\begin{array}{l}\text { Deficiency in access to } \\
\text { health services. }\end{array}$ & $\begin{array}{l}\text { Oil, electricity } \\
\text { generation }\end{array}$ & $\begin{array}{l}\text { Deforestation, sewage } \\
\text { deficiencies. }\end{array}$ & $\begin{array}{c}{[50,52,53,61,122,} \\
123]\end{array}$ \\
\hline Vichada & $\begin{array}{l}\text { Soy, corn, oil palm, } \\
\text { cashew, yucca, rice, } \\
\text { rubber, wood }\end{array}$ & $\begin{array}{l}\text { Ecotourism, sport fishing, } \\
\text { adventure tourism. }\end{array}$ & $\begin{array}{l}\text { Bovine and buffalo } \\
\text { livestock }\end{array}$ & Malnutrition & $\begin{array}{l}\text { Agroindustry (forestry). } \\
\text { Resin distillation }\end{array}$ & Deforestation & {$[50,124,125]$} \\
\hline
\end{tabular}




\subsubsection{Cattle Raising}

Cattle raising is an activity carried out in a large part of the rural territory of Colombia, and it was one of the few economic sectors that showed growth in 2020 [126]. The PND promotes sustainable cattle raising and the development of agricultural production models with the support of technologies that increase their efficiency.

The proposed application possibilities of IoT focused on precision farming are varied. For example, monitoring the heat (when it is ready for mating) and the geographic location of the animals would allow for an increase of their reproductive efficiency, and protect against theft of cattle [127]. Diseases and health problems suffered by animals on farms cause a loss of productivity, and are a threat to public health [128]. In this sense, it would be possible to control animal diseases such as echinococcus [129] or tumors [130] through IoT management and monitoring systems. It will also be possible to help farmers in making decisions regarding livestock, thanks to AI techniques and machine learning (ML), among others [131]. Currently, there are no reports of a large number of IoT projects oriented to cattle farming in Colombia, although work has been done on the control of livestock mobility [132] or on the detection of animals in heat [133]. This utility of IoT will be enhanced with the improved capacity (bps) of 5G, thanks to increased bandwidth and spatial multiplexing, reducing network congestion and overload [127]. In addition, the reduction in energy consumption and the ability to connect a large number of devices make it possible for IoT to play an important role in the wide geographical scenarios where livestock farming is developed [131].

In this sense, 5G will reinforce the communication infrastructure of WSN, being the transport method to send data to the internet, preferably when using the sub-GHz band (700 MHz) in rural areas. Furthermore, and taking into account the high demand for wireless nodes deployed in large geographical areas required for smart farm environments, mMTC is the ideal option in a 5G scenario to serve the development purposes of this economic sector through ICT.

\subsubsection{Tourism}

Before the start of the pandemic caused by the appearance of the SARS-CoV-2 virus (also known as COVID-19), tourism had achieved record figures in Colombia, reaching $4,515,932$ non-resident foreign visitors, growing at a rate greater than the rest of the countries of the region [134]. Most Colombian departments consider tourism a key factor in their development, and for that they have a diverse offer. Although there are traditional tourist proposals for sun and beach (or coastal tourism) mainly in Bolívar, Magdalena, Sucre, and San Andrés-or historical tourism such as Cartagena and Mompox (in the department of Bolívar), Santa Marta (capital of Magdalena), or Popayán (capital of Cauca)—-there are also many other alternatives such as adventure and ecological tourism in Chocó, Caquetá, Vichada, and Tolima with their jungles and natural reserves, while due to their biodiversity, the natural parks and the eastern plains are attractive in the departments of the Andean region and the Orinoco, respectively. In addition, the festivities and events also attract a large number of tourists each year, as in the case of Barranquilla (capital of the department of Atlántico) with its carnival, Medellín (capital of the department of Antioquia) with the Feria de las Flores, Pasto (Capital of the department of Nariño) with the Carnival of Blacks and Whites, or Cali (capital of the department of Valle del Cauca) with its fair. There are also health or medical tourism offers in some cities in Colombia, such as in the case of Barranquilla, Cali, Medellín, or Bucaramanga (capital of Santander). Thanks to the significant offer and investment in infrastructure and human training, these locations have been consolidated as suggested destinations to perform different types of interventions, mainly aesthetic and cardiology specialties [135-137].

The integration of IoT and 5G could provide great benefits to the tourism sector in these regions of Colombia, taking into account that despite its potential, many of these departments of tourism still do not represent a significant percentage of their economy, and therefore, there is a great growth opportunity. In this regard, and considering that the 
tourism industry requires a large amount of information and is increasingly dependent on ICT [138], it is necessary to collect information at all stages of a tourist trip or event, so that appropriate actions can be taken to satisfy customers and improve the competitiveness of this sector. The collection of information through IoT systems would help develop practical applications for the benefit of users such as guided tours, smart shopping, or efficient travel management [138,139], as well as to the monitoring of architectural works in historic buildings with at-risk infrastructure [140]. It would also allow for the implementation of algorithms for predicting user behavior as proposed in [141], or be complemented with big data tools so that tourists have a personalized experience and are able to make decisions about their trips $[142,143]$. It would also help to improve the experience and safety of travelers who make use of extended reality technologies through sensors, as in [144], where they used ultrasound transducers that perceive the surrounding environment, providing complementary information to augmented reality systems. The 5G network plans to offer small-cell and mm-Wave connectivity to tourists at any time and location [145]. Socalled "green, ethical, and clean" tourism will best integrate IoT with 5G, boosting the use of virtual reality, augmented reality, and AI, thanks to the expected high speed and coverage [146].

In the Colombian context, virtual reality and augmented reality are the technologies of current interest in the development of prototypes. For example, in [147], they were used in the promotion of historical sites in Cartagena, or to improve the experience of tourists visiting tourist sites in Bucaramanga [148].

Considering the transmission of large amounts of data, as well as the efficient management of a high density of equipment in small areas due to the high number of tourists traveling for entertainment purposes, meeting in open spaces (beaches, festivals, fairs) or in enclosed areas (museums, historical buildings), and also for health tourism reasons, it is necessary to measure different variables to connect, monitor, and diagnose remotely [149]. Here, the 5G eMBB application scenario is the best option to support the communication needs and information volume in IoT applications in the tourism sector.

\subsubsection{Agriculture}

All DDPs present the intention of strengthening the agricultural sector in each of the Colombian departments. Due to the geographical characteristics of each of them, different crops predominate that could benefit from the interaction of IoT, such as cassava in the Caribbean and Amazon region, coffee in the Andean region, sugarcane in the Pacific region, and rice in the Orinoco region. Despite the hydrological potential of Colombia and the quality of some of its lands for agriculture, this country is regularly affected by droughts that originate from natural phenomena such as the so-called "Niño". In addition, there are many other factors, among which are the appearance of pests, bad practices in crops, and little technification. These factors have an impact on productivity and influence the cost of food, putting food sustainability at risk. For this reason, the Ministry of Agriculture has given recommendations to mitigate the effect of these events, including the monitoring of information and alerts issued by the Institute of Hydrology, Meteorology, and Environmental Studies (IDEAM); an alternate means of water supply; generating alternative mechanisms to shade crops and animals; planning irrigation shifts for access to water; constructing reservoirs for water storage; and generating plans for pest control [150].

IoT offers many options that could help farmers in managing their crops. One such option is the implementation of low-cost automated irrigation systems that use wireless technologies for the purpose of monitoring soil moisture and verifying its operation through IoT [151,152]. It is also attractive to develop control technologies for irrigation or fertigation systems, and to plan the administration of crops, which allows for an increase in the yield of crops and, at the same time, a reduction in environmental risks [153-155]. Another possibility is the monitoring of the crop or of climatological variables to which 
plantation managers can access remotely and through different platforms, allowing for efficient management [156,157].

With the speed of data transfer offered by $5 \mathrm{G}$, agricultural operations have had a positive impact since 2017, helping to improve crop management through the use of drones and/or robots, the real-time monitoring of variables, and data analysis, among many others [158]. Another favorable aspect in this economic sector is the improvements introduced in 5G, by reducing IoT implementation costs and increasing its use to more efficiently integrate the agricultural cycle, i.e., from the sowing stage to the distribution of products [159].

Regarding the Colombian scenario, agriculture is possibly the economic and social sector where most research is being done in relation to IoT applications. For example, in $[160,161]$, IoT was part of the set of tools used in crop irrigation management, while in [162] it improved production in a tomato field. These and other opportunities for implementation in agriculture will be possible with 5G, requiring a great effort from the public and private sectors to promote the implementation of new IoT projects, with the 5G mMTC scenario being the most appropriate because it supports many nodes deployed in large areas of crops. Currently, many rural areas in Colombia do not have access to cell phone networks or to the Internet as a complementary tool in their agricultural activities. To overcome this obstacle, the Colombian government has reached agreements with the main mobile telephone operators of the country, extending the cellular telephone network infrastructure to 3658 new rural locations before the end of 2024. This new technological panorama is favorable for projecting new applications that optimize the processes of agricultural activities. Otherwise, without this wireless coverage, the high costs of implementing IoT projects in agriculture would be low or nonexistent, due to the installation of the infrastructure $[163,164]$.

\subsubsection{Health}

The health problems of the different Colombian departments are diverse. In some departments, among which Norte de Santander, Caquetá, San Andrés y Providencia, Córdoba, and Chocó stand out, there are deficiencies in health services across several of their respective localities that surround them. In addition, due to the extension of their territories and the lack of adequate road infrastructure or geography, timely access to health professionals and medical centers is difficult. For example, in Chocó, a large part of the towns that make up this department are more than $7 \mathrm{~h}$ away from the municipal seats or the capital [112], while in Nariño, $40 \%$ of the total extension of the department does not count with road connection [113], which makes access to doctors, laboratories, or hospitals difficult. Another common health problem in Colombian departments are respiratory, circulatory, and heart diseases, which occur even in more developed departments such as Valle del Cauca, Antioquia, and Santander. Health systems based on IoT and 5G are a great opportunity to improve the quality of services and increase coverage, especially in remote areas of these regions where access to health services can be expensive and slow due to the great distances to travel. For example, the department of Antioquia in its PDD considers that the activities of telemedicine, prehospital telecare, and remote diagnosis, among others, can be effective in improving health care in remote locations [76].

Real-time monitoring applications using body area networks (WBAN) can become indispensable tools, monitoring vital signs of patients located in quarantine areas, in highdemand hospital centers, or in remote locations. The data can then be sent to IoT devices, which then forward them to the treating physician or diagnostic systems using the $5 \mathrm{G}$ network [165-168]. Through IoT, it would also be possible to monitor patients who have travelled hundreds of kilometers to hospital centers, allowing health care workers to know vital parameters such as heartbeat, temperature, or respiratory rate in advance, making possible the provision of an efficient service with less loss of time. Some resources that are a part of an intelligent hospital infrastructure and that will dominate the health scenario in 
the future area include telecare assets, networked medical devices, networks, and sensors distributed in buildings [165].

IoT/5G deployment is currently contributing to the rise of healthcare applications because they leverage higher availability, high scalability, and low network latency [169]. In addition, multi-access perimeter computing nodes in $5 \mathrm{G}$ provide sufficient compute and storage capacities at the edge of the network [170].

Some prototypes of IoT applications in health care have already been implemented in Colombia for the remote monitoring of patients, as in [171], where large data sets were obtained through sensors to perform predictive analysis among a group of people with hypertension problems, and thus designing prevention campaigns; or in [172], oriented to the surveillance of this same disease. Other projects have been oriented to the remote monitoring of patients in hospital centers [173], or even to self-monitoring, in an attempt to avoid traveling to hospitals or clinics [174].

Critical IoT applications in the health sector will be potentiated with the advantages offered by $5 \mathrm{G}$ networks because they demand high reliability and low communication latency, being the uRLLC scenario appropriate for the sensitive registration of vital signs information collected by devices used for patients [165,175-177].

\subsubsection{Industry}

Table 4 shows that manufacturing-type industries predominate in Colombia, mainly in departments with greater economic development such as Antioquia, Atlántico, Cundinamarca, Santander, and Valle del Cauca. The modern industry increasingly requires low latency, high determinism, high bandwidth, and high resilience computing and communication; characteristics necessary to implement fast, intelligent, and autonomous decision-making [178]. In this sense, IoT and 5G are considered technological tools of great potential, because industry 4.0 is increasingly incorporating wireless connections in the manufacturing and logistics of its processes [179]. In addition, an increase is expected in the number of industrial wireless sensors that collect information on environmental conditions and the processes carried out in the plants, allowing for self-diagnosis activities to be carried out for the maintenance and operation of the equipment [180]. There are also opportunities with industrial production robots that can be controlled in the cloud [178], for which 5G will be key in the timely exchange of information through high-speed connections [180].

The positive impact of 5G features on industry offers improved real-time production monitoring or information about the status of a piece of equipment or its manufacturing process [181]. Some projections indicate that the digitalization of the manufacturing industry enabled by $5 \mathrm{G}$ will offer new production opportunities, increasing global manufacturing revenues from USD 52 billion in 2022 to USD 233 billion in 2026 [182].

In Colombia, the Superintendence of Industry and Commerce presented the main aspects to implement IoT in the industrial sector, specifically in logistics, inventory management, and smart factories. It also identified a patent application by a Colombian company on the issue of tracking for the control and traceability of the position and fixed or moving location of tangible objects or merchandise by means of radiofrequency and image recognition [183]. Because industrial services are classified as critical, it is convenient to make use of the uRLLC scenario, so that the probability of failure at low latency is reduced $[184,185]$.

\subsubsection{Environment}

Deforestation is the predominant environmental problem in the regions that make up the Colombian territory. Figures from the Ministry of Environment and Sustainable Development (Minambiente) indicate that in 2020, 171,685 hectares of forest were lost in Colombia as a result of deforestation [186].

Some actions have been carried out with the purpose of finding solutions through IoT that allow reducing deforestation levels with the implementation of technologies that detect illegal logging and the commercialization of wood. Amazon Web Services (AWS), in collaboration with the Jorge Tadeo Lozano University and the United States Embassy, 
sponsored an event in 2019 where they faced challenges to control the production chain, and combatted illegal logging and deforestation. One of the solutions proposed was based on IoT, and consisted of tracking the record from the moment of extraction to the delivery to manufacturers through devices installed in trucks [187]. Also, in [188], the advantage of IoT aimed at preventing illegal deforestation is demonstrated, integrating inclination and sound sensors to detect the poaching of trees. Regarding forest fires, DDPs have identified them as one of the main environmental problems in Colombia, causing serious damage to the biodiversity of the areas where they occur, as well as deterioration in air quality in cities [189]. IoT can also be a part of the solution to this phenomenon; more specifically, its application in this field is a topic widely researched and reported in the literature, and, therefore, it could serve as an example of implementation to present solutions in Colombia. For example, in [190-192], they used IoT devices with meteorological sensors (rain, temperature, humidity, wind speed, $\mathrm{CO}, \mathrm{CO}_{2}$ ) for the detection and prediction of forest fires, respectively, making timely decisions that help to preserve forests and jungles.

There is also research on air pollution and environmental temperature and humidity monitoring that prefers $5 \mathrm{G}$ technology to transmit data over the cloud rather than Zigbee, Bluetooth, and Wi-Fi, because of its high communication range and low power consumption [193].

Regarding 5G, a wide use of this technology is not reported in applications related to the topic discussed in this item. However, if these networks are deployed in the future, it would be possible to develop similar projects in Colombia, especially with the use of bands below $1 \mathrm{GHz}(700 \mathrm{MHz})$ that have been considered for use in rural areas, because they have lower attenuation with respect to other higher frequency bands due to the characteristic of its wavelength. Also, the mMTC 5G scenario is the best option for implementation in IoT applications that monitor environmental variables in the aforementioned areas of study and research.

The application possibilities of IoT based on 5G are wide and generate a high expectation of development in the Colombian economy and society. Moreover, sectors such as education, security, sports, and transportation, among others, could also benefit from the joint implementation of these two technological tools. Figure 4 summarizes the comparative analysis of the possibility of applications for each natural region of Colombia.

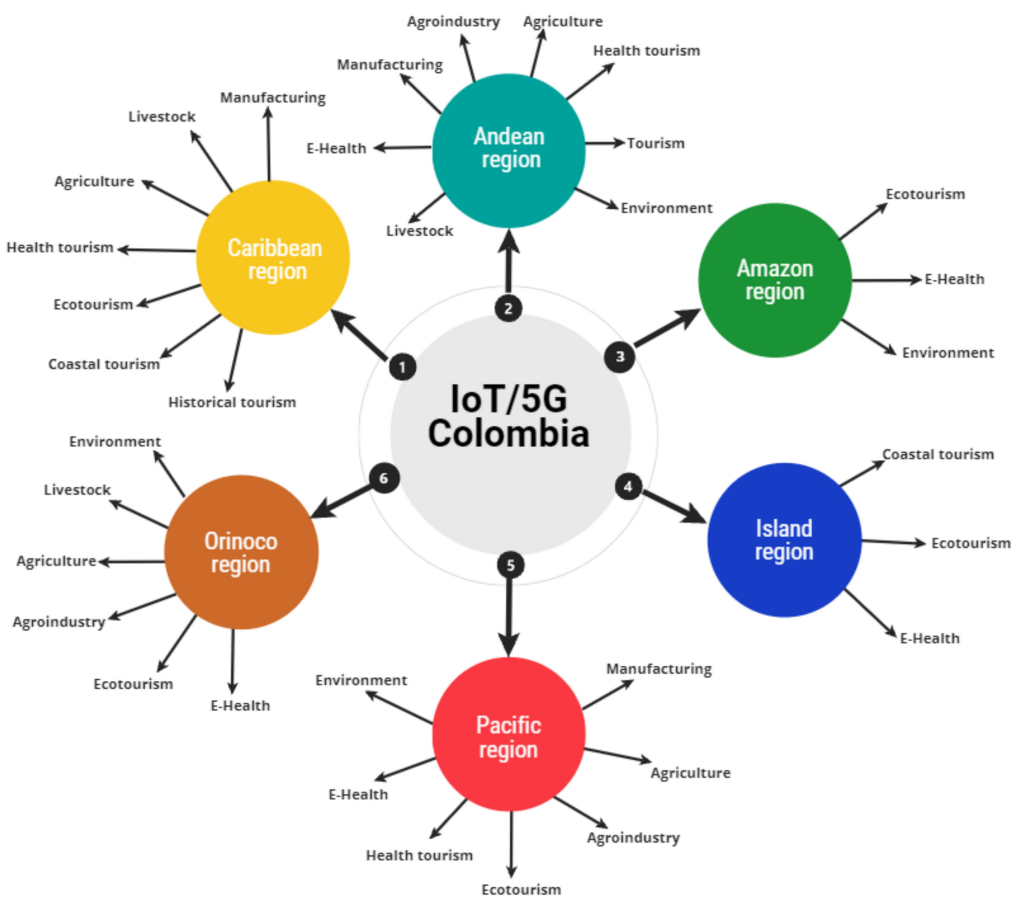

Figure 4. IoT/5G applications potential summary in natural regions of Colombia. 


\section{Discussion}

It is evident that $5 \mathrm{G}$ represents an important evolution in the development of wireless networks passing from focusing on users, to machines, to the industry, and through the cloud, within the Internet of Things. Moreover, changes are observed in the spectrum management with respect to what was done by most nations until the deployment of fourth generation networks, currently considering the use of bands above $6 \mathrm{GHz}$, which brings more bandwidth, but also creates challenges in relation to the propagation of radio waves. However, we believe that all of the advantages offered by $5 \mathrm{G}$ will not be available before September 2022. This is due to the fact that, since $4 \mathrm{G}$ is the base that initially supports it, the latter is still expanding its deployment in most of the rural areas of the Colombian territory, promoted by bilateral agreements between the state and private companies. Therefore, it will be necessary to increase regulatory policies that allow operators to expand their coverage, as well as incentives for users to acquire the necessary services and equipment to implement IoT in different areas of the economy; for example, the current tax exemption for the purchase of smartphones that are below approximately USD 185.

This review shows that there is a wide range of tourism in parks and nature reserves, which, together with agricultural activities, deforestation, and major health issues, are more relevant in rural scenarios. Consequently, the short- and medium-term use of IoT through $5 \mathrm{G}$ as technological tools to help solve challenges in health, environment, agribusiness, and eco-tourism, among others, will depend on different projections and agreements between the state and private enterprise, highlighting:

- early execution of spectrum auction processes;

- incentives for operators to deploy infrastructure in areas where there is no high purchasing power by the population, and little opportunity for rapid return on investment;

- rapid regulation for spectrum below $1 \mathrm{GHz}$ to be auctioned and promptly used in rural areas, to overcome propagation problems and the number of antennas that would have to be installed compared to higher frequency bands; and

- implementation of applications in 5G mMTC and eMBB scenarios to satisfy the high density of equipment per square kilometer, as well as high transmission rate requirements.

As for industrial activities, the results of health tourism and events such as trade fairs and congresses are mainly in large cities. As a result, the following should be considered:

- The spectrum solution to serve IoT applications in these cases should be oriented to bands above $1 \mathrm{GHz}$.

- In cases of applications that demand a high data rate, such as virtual reality and big data for tourism in major urban centers, and machine learning in the industrial sector, eMBB is the scenario that best meets this demand, increasing its channel capacity in the future with the use of millimeter bands.

- uRLLC is the 5G scenario that best meets IoT applications in e-health in hospitals, due to its low latency and better performance in highly mobile equipment.

In the Colombian case, it has been officially established that the first commercial 5G network will be available before the end of August 2022, with progress being made by MinICT in pilot tests and technical regulations.

Finally, the use of IoT applications with 5G described throughout the document is an opportunity to increase the economic and social wealth in Colombia, making viable the execution of future projects associated with the fundamental demands in different geographical scenarios throughout the Colombian territory.

Author Contributions: Conceptualization, A.B.-U.; methodology, D.C.-P. and A.C.-P.; validation, A.C.-P.; formal analysis, A.B.-U., J.M.-B. and J.D.-M.; investigation, A.B.-U., J.M.-B., and J.D.-M.; data curation, A.B.-U., J.M.-B. and J.D.-M.; writing-original draft preparation, A.C.-P.; writing-review and editing, D.C.-P. and A.C.-P.; visualization, A.B.-U., J.M.-B. and J.D.-M.; supervision, A.C.-P.; project administration, D.C.-P. and A.C.-P.; resources, D.C.-P. and A.C.-P. All authors have read and agreed to the published version of the manuscript. 
Funding: This research received fund by the Ibero-American Postgraduate University Association (AUIP).

Institutional Review Board Statement: Not applicable.

Informed Consent Statement: Not applicable.

Conflicts of Interest: The authors declare no conflict of interest.

\section{References}

1. Ericsson. Ericsson Mobility Report. Available online: https://www.ericsson.com/4afa0c/assets/local/mobility-report/ documents /2020/november-2020-ericsson-mobility-report-spanish.pdf (accessed on 8 June 2021).

2. Harborth, D.; Pohl, M. Standardization of 5G mobile networks: A systematic literature review and current developments. Int. J. Stand. Res. 2017, 15, 1-24. [CrossRef]

3. Vera Lopez, A.; Chervyakov, A.; Chance, G.; Verma, S.; Tang, Y. Opportunities and challenges of mmWave NR. IEEE Wirel. Commun. 2019, 26, 4-6. [CrossRef]

4. Painuly, S.; Kohli, P.; Matta, P.; Sharma, S. Advance Applications and Future Challenges of 5G IoT. In Proceedings of the 3rd International Conference on Intelligent Sustainable Systems (ICISS 2020), Thoothukudi, India, 3-5 December 2020; pp. $1381-1384$.

5. MinICT. Colombia Resolution Number 000638 Dated 1 April, 2020; Colombia, 2020; pp. 1-6. Available online: https:/ / mintic.gov. co/portal/715/articles-126447_resolucion_pilotos_5G.pdf (accessed on 10 June 2021).

6. MinICT. MinICT Expects to Have a 5G Auction before the Government Ends. Available online: https://mintic.gov.co/portal/ inicio/Sala-de-prensa/MinTIC-en-los-medios/161584:Mintic-espera-tener-una-subasta-5G-antes-que-termine-el-Gobierno (accessed on 10 June 2021).

7. Lalit, C.; Rabindranath, B. A comprehensive survey on resource management in internet of things. J. Telecommun. Inf. Technol. 2020, 2020, 27-43. [CrossRef]

8. Hui, H.; Shi, Q.; Li, F.; Song, Y.; Yan, J. 5G network-based Internet of Things for demand response in smart grid: A survey on application potential. Appl. Energy 2020, 257, 113972. [CrossRef]

9. Akpakwu, G.A.; Silva, B.J.; Hancke, G.P.; Abu-Mahfouz, A.M. A Survey on 5G Networks for the Internet of Things: Communication Technologies and Challenges. IEEE Access 2017, 6, 3619-3647. [CrossRef]

10. Shafique, K.; Khawaja, B.A.; Sabir, F.; Qazi, S.; Mustaqim, M. Internet of things (IoT) for next-generation smart systems: A review of current challenges, future trends and prospects for emerging 5G-IoT Scenarios. IEEE Access 2020, 8, 23022-23040. [CrossRef]

11. Baek, S.; Kim, D.; Tesanovic, M.; Agiwal, A. 3GPP new radio release 16: Evolution of 5G for industrial internet of things. IEEE Commun. Mag. 2021, 59, 41-47. [CrossRef]

12. Li, S.; Xu, L.D.; Zhao, S. 5G Internet of Things: A survey. J. Ind. Inf. Integr. 2018, 10, 1-9. [CrossRef]

13. ITU. World Radiocommunication Conference (WRC-19)—Final Acts. Available online: https://www.itu.int/dms_pub/itu-r/ opb/act/R-ACT-WRC.14-2019-PDF-S.pdf (accessed on 28 April 2021).

14. Ramesh, M.; Priya, C.G.; Ananthakirupa, V.P.M.B.A.A. Design of efficient massive MIMO for 5G systems-Present and past: A review. In Proceedings of the 2017 International Conference on Intelligent Computing and Control (I2C2), Coimbatore, India, 23-24 June 2017; pp. 1-4.

15. ETSI. Digital Cellular Telecommunications System (Phase 2+) (GSM); Universal Mobile Telecommunications System (UMTS); LTE; 5G, Release 15 (3GPP TR 21.915 version 15.0.0 Release 15); ETSI: Côte d'Azur, France, 2019; pp. 1-120.

16. ITU. Laying the Groundwork for 5G: Opportunities and Challenges; ITU: Geneva, Switzerland, 2018.

17. Cama-Pinto, D.; Damas, M.; Holgado-Terriza, J.A.; Gómez-Mula, F.; Calderín-Curtidor, A.C.; Martínez-Lao, J.A.; Cama-Pinto, A 5G Mobile Phone Network Introduction in Colombia. Electronics 2021, 10, 922. [CrossRef]

18. Bechta, K.; Ziólkowski, C.; Kelner, J.M.; Nowosielski, L. Modeling of Downlink Interference in Massive MIMO 5G Macro-Cell. Sensors 2021, 21, 597. [CrossRef] [PubMed]

19. FCC. FCC Announces Winning Bidders in C-Band Auction. Available online: https://www.fcc.gov/document/fcc-announceswinning-bidders-c-band-auction (accessed on 6 April 2021).

20. FCC. America's 5G Future. Available online: https:/ /www.fcc.gov/5G (accessed on 6 April 2021).

21. Ministerio de Economía y Empresa. Nota Informativa sobre la Subasta de Espectro Banda 3600-3800 MHz. Available online: https:/ / avancedigital.mineco.gob.es/5G/Documents/NOTA-INFORMATIVA-SUBASTA-3600-3800-MHz.pdf (accessed on 6 April 2021).

22. European 5G Observatory. The 5G Greek Auction Raised 372.3 Million EUR. Available online: https://5gobservatory.eu/the-5ggreek-auction-raised-372-3-million-eur/ (accessed on 6 April 2021).

23. PTS. The Auctions in the $3.5 \mathrm{GHz}$ and $2.3 \mathrm{GHz}$ Bands Are Concluded. Available online: https://www.pts.se/en/news/pressreleases/2021/the-auctions-in-the-3.5-ghz-and-2.3-ghz-bands-are-concluded/ (accessed on 6 April 2021).

24. Ofcom. Award of $700 \mathrm{MHz}$ and 3.6-3.8 GHz Spectrum by Auction. Available online: https://www.ofcom.org.uk/spectrum/ spectrum-management/spectrum-awards/awards-in-progress/700-mhz-and-3.6-3.8-ghz-auction (accessed on 6 May 2021).

25. European 5G Observatory. Japan Assigns 5G Spectrum to Four Operators. Available online: https://5gobservatory.eu/japanassigns-5g-spectrum-to-four-operators/ (accessed on 6 May 2021).

26. MinICT. Colombia Plan 5G Colombia-The Digital Future Belongs to Everyone. 2019. Available online: https://mintic.gov.co/ micrositios/plan_5g//764/articles-162230_recurso_1.pdf (accessed on 6 May 2021). 
27. National Spectrum Agency. National Frequency Band Allocation Table; National Spectrum Agency: Bogota, Colombia, $2021 ;$ p. 359.

28. MinICT. Colombia Framework Plan for the Allocation of Spectrum Use Permits 2020-2022; MinICT: Bogota, Colombia, 2020.

29. MinICT. Colombia Resolution Number 001322 of July 27, 2020; MINTIC Colombia: Bogota, Colombia, 2020; pp. 1-2.

30. Communications Industry Directorate of MinICT Allocation Report-Spectrum for 5G Technical Test Use. 2020. Available online: https://mintic.gov.co/portal/715/articles-151433_recurso_1.pdf (accessed on 6 May 2021).

31. Claro. Claro Starts New 5G Trials in Several Cities. Available online: https://www.claro.com.co/institucional/pruebas-5g/ (accessed on 18 March 2021).

32. Military Hospital HOMIL and Movistar Present Second 5G Pilot. Available online: https://www.hospitalmilitar.gov.co/index. php?idcategoria=69906 (accessed on 19 March 2021).

33. TigoUne 5G Tests: TigoUne Obtained the Highest Mobile Speed Ever Achieved in Colombia. Available online: http: / / saladeprensa.une.com.co/index.php/1945-pruebas-5g-tigoune-obtuvo-la-mayor-velocidad-movil-jamas-alcanzada-encolombia (accessed on 18 March 2021).

34. Claro. 5G Technology in Colombia: We Are Already in Trials. Available online: https://www.claro.com.co/empresas/sectores/ noticias-interes/5g-colombia/ (accessed on 1 April 2021).

35. MinICT. Colombia Preliminary Analysis of the Objective Selection Process for the Allocation of Spectrum Use Permits in IMT Bands. 2020. Available online: https://mintic.gov.co/portal/715/articles-146624_resolucion_1322_20200727_soporte_tecnico.pdf (accessed on 2 April 2021).

36. MinICT. By May 2021, 954 Rural Localities Will Have 4G Mobile Service, Announces Minister Karen Abudinen. Available online: https:/ / mintic.gov.co/portal/inicio/Sala-de-Prensa/Noticias/161329:En-mayo-de-2021-954-localidades-de-zonas-ruralestendran-servicio-movil-4G-anuncia-la-ministra-Karen-Abudinen (accessed on 3 October 2021).

37. MinICT. Colombia Transition to New Technologies Plan. 2020. Available online: https://mintic.gov.co/portal/715/articles-1455 50_plan_transicion_nuevas_tecnologias_20200624a.pdf (accessed on 3 October 2021).

38. GSMA. 5G Spectrum-GSMA Public Policy Position. 2021. Available online: https://www.gsma.com/spectrum/wp-content/ uploads/2021/04/5G-Spectrum-Positions.pdf (accessed on 15 June 2021).

39. 5G Americas. 5G Spectrum Vision. 2019. Available online: https://www.5gamericas.org/wp-content/uploads/2019/07/5G_ Americas_5G_Spectrum_Vision_Whitepaper-1.pdf (accessed on 4 April 2021).

40. Huawei 5G Spectrum —Public Policy Position; Huawei: Shenzhen, China, 2020.

41. Cisco. Cisco Annual Internet Report. 2020. Available online: https://www.cisco.com/c/en/us/solutions/collateral/executiveperspectives/annual-internet-report/white-paper-c11-741490.pdf (accessed on 4 April 2021).

42. DG Communications Networks, Content \& Thecnology. Identification and Quantification of Key Socio-Economic Data to Support Strategic Planning for the Introduction of 5G in Europe. 2016. Available online: https://op.europa.eu/en/publication-detail/-/ publication/2baf523f-edcc-11e6-ad7c-01aa75ed71a1/language-en (accessed on 29 March 2021).

43. Zhang, L.; Liang, Y.; Xiao, M. Spectrum Sharing for Internet of Things: A Survey. IEEE Wirel. Commun. 2019, 26, 132-139. [CrossRef]

44. National Planning Department. What Is the National Development Plan? Available online: https://www.dnp.gov.co/DNPN/ Paginas/Que-es-el-Plan-Nacional-de-Desarrollo.aspx (accessed on 13 June 2021).

45. National Planning Department. National Development Plan-Pact for Colombia, Pact for Equity; Colombia. 2019. Available online: https: / / colaboracion.dnp.gov.co/CDT/Prensa/Resumen-PND2018-2022-final.pdf (accessed on 8 August 2021).

46. National Planning Department. Territorial Development Plans. Available online: https://pazvictimas.dnp.gov.co/Paz-conenfoque-territorial/Paginas/pdt.aspx (accessed on 13 June 2021).

47. Government of the Atlántico. Development Plan 2020-2023 “Atlántico for the People". Available online: https: / www.atlantico. gov.co/images/stories/plan_desarrollo/PlanDesarrollo_2020-2023-Definitivo-A1.pdf (accessed on 5 May 2021).

48. AGRONET Atlántico. Main Crops by Area Planted in 2017. 2018. Available online: https://www.agronet.gov.co/Documents/ ATL\%C3\%81NTICO_2017.pdf (accessed on 3 August 2021).

49. SITUR Atlántico. Main Reason for Travel to the Department of Atlántico. Available online: https://www.situratlantico.com/ indicadores / receptor (accessed on 25 June 2021).

50. Directorate of Fishery and Aquaculture Chain Management. Bovine-Meat Chain. 2020. Available online: https://sioc. minagricultura.gov.co/Bovina/Documentos/2020-12-30\%20Cifras\%20Sectoriales.pdf (accessed on 3 August 2021).

51. Directorate of Fishery and Aquaculture Chain Management. Pork Meat Chain. 2019. Available online: https://sioc. minagricultura.gov.co/Porcina/Documentos/2020-06-30\%20Cifras\%20Sectoriales.pdf (accessed on 3 August 2021).

52. Directorate of Fishery and Aquaculture Chain Management. Poultry Chain. 2019. Available online: https://sioc.minagricultura. gov.co/Avicola/Documentos/2020-03-30\%20Cifras\%20Sectoriales.pdf (accessed on 3 August 2021).

53. Directorate of Fishery and Aquaculture Chain Management. Aquaculture Chain. 2020. Available online: https://sioc. minagricultura.gov.co/Acuicultura/Documentos/2020-12-30\%20Cifras\%20Sectoriales.pdf (accessed on 3 August 2021).

54. Directorate of Fishery and Aquaculture Chain Management. Ovino-Caprina Chain. 2019. Available online: https://sioc. minagricultura.gov.co/OvinoCaprina/Documentos/2019-03-31\%20Cifras\%20Sectoriales.pdf (accessed on 3 August 2021).

55. ACOLGEN. Map of Electricity Generation in Colombia. Available online: https://www.acolgen.org.co/mapa-generacion/ (accessed on 3 August 2021). 
56. Superintendence of Transportation Port Traffic in Colombia. 2021. Available online: https://www.supertransporte.gov.co/ documentos/2021/Febrero/Puertos_04/BOLETIN-TRAFICO-PORTUARIO-2020.pdf (accessed on 3 August 2021).

57. Office of Economic Studies Manufacturing Sector Report. 2021. Available online: https://www.mincit.gov.co/getattachment/ estudios-economicos/estadisticas-e-informes/informes-de-industria/2021/enero/oee-dp-industria-manufacturera-enero-20 21.pdf.aspx (accessed on 3 August 2021).

58. Government of La Guajira. Departmental Development Plan of La Guajira "United for Change 2020-2023". Available online: https: / / www.laguajira.gov.co/NuestraGestion/PlaneacionGestionyControl/PDM\%202020.pdf (accessed on 3 August 2021).

59. AGRONET La Guajira. Main Crops by Area Planted in 2017. 2017. Available online: https://www.agronet.gov.co/Documents / LA\%20GUAJIRA_2017.pdf (accessed on 3 August 2021).

60. Colombia Tourist Information Center National Statistics-National Natural Parks. 2021. Available online: https:/ / citur.gov.co/ estadisticas /df_parques_naturales/detalle/18?t=1\#gsc.tab=0 (accessed on 3 August 2021).

61. Directorate of Fishery and Aquaculture Chain Management. Equine, Donkey and Mule Chain. 2019. Available online: https: //sioc.minagricultura.gov.co/Equino/Documentos/2019-03-30\%20Cifras\%20Sectoriales.pdf (accessed on 3 August 2021).

62. Government of Bolívar. Development Plan for the Department of Bolivar 2020-2023 “Bolivar First". Available online: https: / / www.asambleadebolivar.gov.co/servicios/2020/proyecto-de-ordenanza-plan-de-desarrollo-bolivar-2020-2023 (accessed on 18 June 2021).

63. AGRONET Bolivar. Main Crops by Area Planted in 2017. 2018. Available online: https://www.agronet.gov.co/Documents / BOL\%C3\%8DVAR_2017.pdf (accessed on 3 August 2021).

64. SITUR Bolívar. Sites of Interest. Available online: http:/ / www.siturbolivar.com/studies (accessed on 24 June 2021).

65. Directorate of Fishery and Aquaculture Chain Management. Bee and Beekeeping Chain. 2019. Available online: https: / / sioc.minagricultura.gov.co/Apicola/Documentos/2019-03-30\%20Cifras\%20sectoriales.pdf (accessed on 3 August 2021).

66. Government of Cesar. Departmental Development Plan 2020-2023 “We Do It Better”. Available online: http://cesar.gov.co/d/ filesmain/plan_desarrollo/plan_de_desarrollo_2020_2023_lo_hacemos_mejor_act.pdf (accessed on 16 June 2021).

67. AGRONET Cesar. Main Crops by Area Planted in 2017. 2018. Available online: https://www.agronet.gov.co/Documents/ CESAR_2017.pdf (accessed on 3 August 2021).

68. Government of Cesar. Tourist Guide of the Department of Cesar. Available online: http://cesar.gov.co/d/index.php/es/ mainmeneldpto/mengobtur (accessed on 23 June 2021).

69. Government of Córdoba. Departmental Development Plan “Now It's Córdoba's Turn 2020-2023". Available online: https:/ /gobcordoba.micolombiadigital.gov.co/sites/gobcordoba/content/files / 000057/2828_ordenanza-0009-plan-dedesarrollo-departamental--20202023--ahora-le-toca-a-cordoba--web.pdf (accessed on 21 June 2021).

70. AGRONET Córdoba. Main Crops by Planted Area in 2017. 2018. Available online: https:/ / www.agronet.gov.co/Documents/C\% C3\%93RDOBA_2017.pdf (accessed on 3 August 2021).

71. Government of Magdalena. Departmental Development Plan “Magdalena Renace 2020-2023”. Available online: https: / / magdalena.micolombiadigital.gov.co/sites/magdalena/content/files/000782/39075_plan-de-desarrollo-departamentalmagdalena-renace-2020--2023-1.pdf (accessed on 21 June 2021).

72. AGRONET Magdalena. Main Crops by Planted Area in 2017. 2018. Available online: https://www.agronet.gov.co/Documents/ MAGDALENA_2017.pdf (accessed on 3 August 2021).

73. SITUR Magdalena. Attractions. Available online: https://www.siturmagdalena.com/quehacer/index?tipo=2 (accessed on 22 June 2021).

74. Government of Sucre. Departmental Development Plan Sucre 2020-2023 “Sucre Diferente". Available online: https: / / sucre.micolombiadigital.gov.co/sites/sucre/content/ files/000833/41615_proyecto-de-ordenanza-por-la-cual-se-adopta-elplan-departamental.pdf (accessed on 20 June 2021).

75. AGRONET Sucre. Main Crops by Sembled Area. 2018. Available online: https://www.agronet.gov.co/Documents/SUCRE_2017 .pdf (accessed on 3 August 2021).

76. Government of Antioquia. Development Plan “United for Life 2020-2023”. Available online: https:/ / plandesarrollo.antioquia gov.co/archivo/PlanDesarrolloUNIDOS_VF-comprimido-min.pdf (accessed on 1 June 2021).

77. Agricultural and Forestry Chain Directorate. Flower Chain. 2020. Available online: https://sioc.minagricultura.gov.co/Flores/ Documentos / 2019-12-30\%20Cifras\%20Sectoriales.pdf (accessed on 3 August 2021).

78. AGRONET Antioquia. Main Crops by Area Planted in 2017. 2018. Available online: https://www.agronet.gov.co/Documents/ ANTIOQUIA_2017.pdf (accessed on 3 August 2021).

79. Government of Boyacá. Development Plan 2020-2023 “Social Pact for Boyacá: Land That Continues to Advance”. Available online: https:/ / www.boyaca.gov.co/wp-content/uploads/2020/06/pdd2020-2023boy.pdf (accessed on 23 May 2021).

80. Boyacá Tourism Information System. Tourist Map of Boyacá. Available online: https://situr.boyaca.gov.co/boyaca-es-paravivirla/\# (accessed on 25 June 2021).

81. Government of Caldas. Development Plan 2020-2023 “United Is Possible”. Available online: https:/ / docs.google.com/viewer? url=https: / / caldas.gov.co/index.php/inicio/mecanismos-de-control/transparencia-1/1494-plan-de-desarrollo-de-caldas-20 20-2023/download?p=1 (accessed on 20 May 2021).

82. AGRONET Caldas. Main Crops by Area Planted in 2017. Available online: https://www.agronet.gov.co/Documents/CALDAS_ 2017.pdf (accessed on 24 June 2021). 
83. Government of Cundinamarca. Departmental Development Plan 2020-2024 "Cundinamarca, Region That Progresses". Available online: http:/ / www.cundinamarca.gov.co/wcm/connect/37b90ffc-f445-462b-8faa-8a16f4427fe8/PLAN+DE+DESARROLLO+ PLIEGOS1_compressed.pdf?MOD=AJPERES\&CVID=njCfayi\&CVID=njCfayi\&CVID=njCfayi\&CVID=njCfayi\&CVID=njCfayi (accessed on 19 May 2021).

84. Government of Huila. Departmental Development Plan 2020-2023 “Huila Crece”. Available online: https:/ /www.huila.gov.co/ documentos/1336/plan-de-desarrollo-2020-2023/ (accessed on 26 June 2021).

85. AGRONET Huila. Main Crops by Sembled Area 2017. Available online: https://www.agronet.gov.co/Documents/HUILA_2017 .pdf (accessed on 3 August 2021).

86. Government of Norte de Santander. Development Plan for Norte de Santander 2020-2023. Available online: http://www nortedesantander.gov.co/Portals/0/PDDNdS2020-2023(Ordenanza006de2020).pdf (accessed on 25 June 2021).

87. Government of Norte de Santander. Tourism. Available online: http:/ /www.nortedesantander.gov.co/Gobernacion/NuestroDepartamento/Turismo (accessed on 24 June 2021).

88. Government of Quindio. Departmental Development Plan 2020-2023. Available online: https://www.quindio.gov.co/home/ docs/items/item_100/PDD_2020_2023_TU_Y_YO_SOMOS_QUINDIO/PDD_QUINDIO_2020_2023_ORDENANZA_002_ .pdf (accessed on 24 June 2021).

89. AGRONET Quindio. Main Crops by Sembled Area 2017. 2018. Available online: https://www.agronet.gov.co/Documents / QUIND\%C3\%8DO_2017.pdf (accessed on 3 August 2021).

90. Government of Quindio. Tourism in the Department of Quindio. Available online: https://www.quindio.gov.co/inicio-turismo (accessed on 25 June 2021).

91. Government of Risaralda. Departmental Development Plan 2020-2023 “Risaralda, Feeling of All”. Available online: https: / / www.risaralda.gov.co/documentos/150205/documento-plan-de-desarrollo/ (accessed on 27 June 2021).

92. AGRONET Risaralda. Main Crops by Area Planted in 2017. 2018. Available online: https://www.agronet.gov.co/Documents/ RISARALDA_2017.pdf (accessed on 3 August 2021).

93. Government of Santander. Departmental Development Plan "Santander Always with You and for the World" 2020-2023. Available online: https:/ / www.santander.gov.co/index.php/documentos-planeacion/send/2200-plan-de-desarrollo-2020-2 023/18585-pdd-2020-2023-completo-pts-nna-aprobado (accessed on 27 June 2021).

94. AGRONET Santander. Main Crops by Area Planted in 2017. Available online: https://www.agronet.gov.co/Documents/ SANTANDER_2017.pdf (accessed on 25 June 2021).

95. SITUR Santander. Main Reason for Travel. Available online: http:/ /www.sitursantander.co/Estadisticas/Graficos (accessed on 25 June 2021).

96. Government of Tolima. Development Plan "El Tolima Unites Us" 2020-2023. Available online: https:/ / regioncentralrape.gov.co/ wp-content/uploads/2020/05/Ordenanza-Plan-de-desarrollo-version-8.pdf (accessed on 27 June 2021).

97. AGRONET Tolima. Main Crops by Area Planted in 2017. 2018. Available online: https://www.agronet.gov.co/Documents/ TOLIMA_2017.pdf (accessed on 3 August 2021).

98. Government of Tolima. Tourism. Available online: https://www.tolima.gov.co/tolima/informacion-general/turismo (accessed on 26 June 2021).

99. Government of Amazonas. Departmental Development Plan 2020-2023 “Progressing with Development". Available online: http: / / www.amazonas.gov.co/noticias/plan-de-desarrollo-departamental-del-amazonas-20202023 (accessed on 22 June 2021).

100. AGRONET Amazonas. Main Crops by Area Planted in 2017. 2018. Available online: https://www.agronet.gov.co/Documents/ AMAZONAS_2017.pdf (accessed on 3 August 2021).

101. Government of Caquetá. Caquetá Departmental Development Plan. Available online: https://caqueta.micolombiadigital.gov.co/ sites / caqueta / content/ files /001016/50760_pdd-caqueta-20202023.pdf (accessed on 22 June 2021).

102. Government of Guainía. Departmental Development Plan 2020-2023 “Guainía Opportunity for All”. Available online: https: //guainia.micolombiadigital.gov.co/sites/guainia/content/files/000550/27454_documento-final-guainia-va1.pdf (accessed on 28 June 2021).

103. Government of Guaviare. Departmental Development Plan 2020-2023 "Solutions at Your Service". Available online: https: //guaviare.micolombiadigital.gov.co/sites/guaviare/content/files/000705/35240_ordenanza-no-410-de-2020.pdf (accessed on 24 June 2021).

104. AGRONET Guaviare. Main Crops by Areas Planted in 2017. 2018. Available online: https://www.agronet.gov.co/Documents / GUAVIARE_2017.pdf (accessed on 3 August 2021).

105. Government of Guaviare. Tourism. Available online: http:/ /www.guaviare.gov.co/tema/turismo/sitios-para-visitar (accessed on 26 June 2021).

106. Government of Putumayo. Putumayo Departmental Development Plan 2020-2023 “Thirteen Municipalities, One Heart”. Available online: https: / / www.putumayo.gov.co/index.php?option=com_content\&view=article\&id=37 (accessed on 23 June 2021).

107. AGRONET Putumayo. Main Crops by Area Planted in 2017. 2018. Available online: https://www.agronet.gov.co/Documents/ PUTUMAYO_2017.pdf (accessed on 3 August 2021).

108. Government of Vaupés. Departmental Development Plan “Vaupés, Together We Can” 2020-2023. Available online: https: / / vaupes.micolombiadigital.gov.co/sites/vaupes/content/files/000483/24103_pdd-vaupes-juntos-podemos-20202023.pdf (accessed on 24 June 2021). 
109. Government of San Andres. Providencia and Santa Catalina Departmental Development Plan San Andres, Providencia and Santa Catalina Islands 2020-2023 “All for a New Beginning". Available online: https:/ / www.sanandres.gov.co/index.php/gestion/ planeacion/plan-de-desarollo/plan-de-desarrollo-anuales/11524-plan-de-desarrollo-todos-por-un-nuevo-comienzo-2020-2 023/ file (accessed on 23 June 2021).

110. Government of Cauca. Departmental Development Plan 2020-2023 “42 Motives to Advance". Available online: https: / / www.cauca. gov.co/NuestraGestion/PlaneacionGestionyControl/PlandeDesarrolloDepartamental2020-2023.pdf (accessed on 1 May 2021).

111. AGRONET Cauca. Main Crops by Area Planted in 2017. Available online: https://www.agronet.gov.co/Documents/CAUCA_ 2017.pdf (accessed on 28 April 2021).

112. Government of Chocó. Departmental Development Plan “Chocó Generating Confidence" 2020-2023. Available online: https: / / choco.micolombiadigital.gov.co/sites/choco/content/files/000440/21975_plan-de-desarrollo-departamental-delchoco-20202023--version-final.pdf (accessed on 27 April 2021).

113. Government of Nariño. Departmental Development Plan "My Nariño, in Defense of What Is Ours" 2020-2023. Available online: https:/ / sitio.narino.gov.co/wp-content/uploads/2020/11/Plan_de_Desarrollo_Mi_Narino_en_Defensa_de_lo_Nuestro_ 2020-2023.pdf (accessed on 6 May 2021).

114. AGRONET Nariño. Main Crops by Areas Planted in 2017. Available online: https://www.agronet.gov.co/Documents/NARI NO_2017.pdf (accessed on 1 May 2021).

115. SITUR Nariño. Tourist Attractions. Available online: https:/ / situr.narino.gov.co/atractivos-turisticos (accessed on 26 June 2021 ).

116. Government of Valle del Cauca. Development Plan “Invincible Valle" 2020-2023. Available online: https:/ / www.valledelcauca. gov.co/loader.php?1Servicio=Tools2\&lTipo=viewpdf\&id=48214 (accessed on 29 April 2021).

117. AGRONET Valle del Cauca. Main Crops by Areas Planted in 2017. 2018. Available online: https://www.agronet.gov.co/ Documents/VALLE\%20DEL\%20CAUCA_2017.pdf (accessed on 3 August 2021).

118. Government of Arauca. Arauca Departmental Participative Development Plan "Building the Future" 2020-2023. Available online: https: / / www.arauca.gov.co/mapa-red-hospitalaria-del-departamento-de-arauca/62-plan-de-desarrollo/2825-plande-desarrollo-departamental-2020-2023 (accessed on 7 May 2021).

119. AGRONET Arauca. Main Crops by Areas Planted in 2017. 2018. Available online: https://www.agronet.gov.co/Documents/ ARAUCA_2017.pdf (accessed on 3 August 2021).

120. Government of Casanare. Development Plan 2020-2023. It Is Time for Productive, Equitable and Sustainable Casanare. Available online: https: / / www.casanare.gov.co/NuestraGestion/PlaneacionGestionyControl/ORDENANZA\%20002-2020\%20PLAN\% 20DE\%20DESARROLLO.pdf (accessed on 5 May 2021).

121. AGRONET Casanare. Main Crops by Areas Planted in 2017. 2018. Available online: https://www.agronet.gov.co/Documents/ CASANARE_2017.pdf (accessed on 3 August 2021).

122. Government of Meta. Departmental Economic and Social Development Plan “Let's Make Meta Great" 2020-2023. Available online: https:/ / asambleameta.micolombiadigital.gov.co/sites/asambleameta/content/files/000189/9449_ordenanza-1069-demayo-30-de-2020.pdf (accessed on 24 April 2021).

123. AGRONET Meta. Main Crops by Area Planted in 2017. 2018. Available online: https://www.agronet.gov.co/Documents/ META_2017.pdf (accessed on 3 August 2021).

124. Government of Vichada. Departmental Development Plan "Work for All of Vichada" 2020-2023. Available online: https:// vichada.micolombiadigital.gov.co/sites/vichada/content/files/000331/16528_plan-de-desarrollo-aprobado-ordenanza.pdf (accessed on 30 April 2021).

125. AGRONET Vichada. Main Crops by Area Planted in 2017. 2018. Available online: https://www.agronet.gov.co/Documents/ VICHADA_2017.pdf (accessed on 3 August 2021).

126. DANE Technical Bulletin GDP IV Quarter 2020; Bogota. 2021. Available online: https://www.dane.gov.co/files/investigaciones/ boletines/pib/bol_PIB_IVtrim20_producion_y_gasto.pdf (accessed on 3 August 2021).

127. Maroto-Molina, F.; Navarro-García, J.; Príncipe-Aguirre, K.; Gómez-Maqueda, I.; Guerrero-Ginel, J.; Garrido-Varo, A.; PérezMarín, D. A Low-Cost IoT-Based System to Monitor the Location of a Whole Herd. Sensors 2019, 19, 2298. [CrossRef] [PubMed]

128. Gattani, A.; Singh, S.V.; Agrawal, A.; Khan, M.; Singh, P. Recent progress in electrochemical biosensors as point of care diagnostics in livestock health. Anal. Biochem. 2019, 579, 25-34. [CrossRef]

129. Yang, S.J.; Xiao, N.; Li, J.Z.; Feng, Y.; Ma, J.Y.; Quzhen, G.S.; Yu, Q. A remote management system for control and surveillance of echinococcosis: Design and implementation based on internet of things. Infect. Dis. Poverty 2021, 10, 50. [CrossRef]

130. Raj, R.; Kola, K.; Bojja, P.; Raja, P. Optimal Technique of Tumor Detection and Prediction of Livestock by Deep Neural Network with TensorFlow and Keras. In Proceedings of the International Conference of Modern Applications on Information and Communication Technology (ICMAICT), Baghdad, Iraq, 8-9 December 2021; Volume 1804.

131. Akhigbe, B.I.; Munir, K.; Akinade, O.; Akanbi, L.; Oyedele, L.O. IoT Technologies for Livestock Management: A Review of Present Status, Opportunities, and Future Trends. Big Data Cogn. Comput. 2021, 5, 10. [CrossRef]

132. Quintero, J. IoT Device for the Control of Livestock Identification and Mobility; National University of Colombia: Bogota, Colombia, 2020.

133. Ariza-Colpas, P.; Morales-Ortega, R.; Piñeres-Melo, M.; Melendez-Pertuz, F.; Serrano-Torne, G.; Hernandez-Sanches, G.; MartínezOsorio, H. Teleagro: IOT Applications for the Georeferencing and Detection of Zeal in Cattle. In Proceedings of the 18th International Conference, CISIM 2019, Belgrade, Serbia, 19-21 September 2019; pp. 232-239. 
134. Ministry of Commerce. 2019 Tourism in Colombia Breaks Records. Available online: https://www.mincit.gov.co/prensa/ noticias/turismo/en-2019-el-turismo-en-colombia-rompio-records (accessed on 28 June 2021).

135. Alcaldía de Santiago de Cali. Turismo de Salud. Available online: https://www.cali.gov.co/turismo/publicaciones/139222 /turismo-de-salud-/ (accessed on 2 May 2021).

136. ANDI. Colombia: Regional Leader in the Export of Healthcare Services. Available online: http://www.andi.com.co/Home/ Noticia/7356-colombia-lider-regional-en-la-exportacio (accessed on 28 June 2021).

137. Mayor's Office of Barranquilla. The Health Cluster, a New Reality That Potentiates Barranquilla's Image to the World. Available online: https: / / www.barranquilla.gov.co/salud/el-cluster-en-salud-nueva-realidad-que-potencializa-al-mundo-la-imagende-barranquilla (accessed on 28 June 2021).

138. Wang, W.; Kumar, N.; Chen, J.; Gong, Z.; Kong, X.; Wei, W.; Gao, H. Realizing the Potential of the Internet of Things for Smart Tourism with 5G and AI Smart Tourism. IEEE Netw. 2020, 34, 295-301. [CrossRef]

139. Sharma, S.; Rishi, O.; Sharma, A. IoTeST: IoT-Enabled Smart Tourism—Shaping the Future of Tourism. In Rising Threats in Expert Applications and Solutions; Springer: Singapore, 2020; pp. 569-576, ISBN 978-981-15-6014-9.

140. Lerario, A.; Varasano, A. An IoT Smart Infrastructure for S. Domenico Church in Matera's "Sassi": A Multiscale Perspective to Built Heritage Conservation. Sustainability 2020, 12, 6553. [CrossRef]

141. Peng, R.; Lou, Y.; Kadoch, M.; Cheriet, M. A human-guided machine learning approach for 5g smart tourism iot. Electronics 2020, 9, 947. [CrossRef]

142. Gao, H. Big Data Development of Tourism Resources Based on 5G Network and Internet of Things System. Microprocess. Microsyst. 2021, 80, 103567. [CrossRef]

143. Kachniewska, M. Smart Tourism: Towards the Concept of a Data-Based Travel Experience. In Handbook of Sustainable Development and Leisure Services; Springer: Cham, Switzerland, 2021; pp. 289-302, ISBN 978-3-030-59819-8.

144. Davoli, L.; Paraskevopoulos, I.; Campanella, C.; Bauro, S.; Vio, T.; Abrardo, A.; Ferrari, G. Ultrasonic-based environmental perception for mobile $5 \mathrm{~g}$-oriented $\mathrm{xr}$ applications. Sensors 2021, 21, 1329. [CrossRef]

145. Siriwardhana, Y.; De Alwis, C.; Gur, G.; Ylianttila, M.; Liyanage, M. The Fight against the COVID-19 Pandemic with 5G Technologies. IEEE Eng. Manag. Rev. 2020, 48, 72-84. [CrossRef]

146. Al-Maroof, R.S.; Akour, I.; Aljanada, R.; Alfaisal, A.M.; Alfaisal, R.M.; Aburayya, A.; Salloum, S.A. Acceptance determinants of 5G services. Int. J. Data Netw. Sci. 2021, 5, 613-628. [CrossRef]

147. Mendoza, R.; Cabarcas, A.; Arnedo, B. Mixed Reality to Promote Cultural Tourism in La Merced Cloister in Cartagena Colombia. In Advances in Tourism, Technology and Systems; Smart Innovation, Systems and Technologies Series; Springer: Cartagena, Colombia, 2021; Volume 209.

148. Pereira, J.; Díaz, J. Prototype of a Tourist Mobile Application for the City of Bucaramanga Implementing Augmented Reality; Universidad Autónoma de Bucaramanga: Bucaramanga, Colombia, 2020.

149. Psiha, M.; Vlamos, P. IoT Applications with 5G Connectivity in Medical Tourism Sector Management: Third-Party Service Scenarios. Adv. Exp. Med. Biol. 2017, 989, 141-154. [CrossRef]

150. AGRONET. Low Productivity in the Land and Appearance of Pests May Be Effects of El Niño. 2018. Available online: https: / / www.agronet.gov.co/Noticias/Paginas/Baja-productividad-en-la-tierra-y-aparici\%C3\%B3n-de-plagas-pueden-serefectos-del-ni\%C3\%B1o.aspx (accessed on 2 May 2021).

151. Mateos Matilla, D.; Lozano Murciego, Á.; Jimenez Bravo, D.M.; Sales Mendes, A.; Quietinho Leithardt, V.R. Low cost center pivot irrigation monitoring systems based on IoT and LoRaWAN technologies. In Proceedings of the 2020 IEEE International Workshop on Metrology for Agriculture and Forestry (MetroAgriFor 2020), Trento, Italy, 4-6 November 2020; pp. $262-267$.

152. Nawandar, N.K.; Satpute, V.R. IoT based low cost and intelligent module for smart irrigation system. Comput. Electron. Agric. 2019, 162, 979-990. [CrossRef]

153. Lin, N.; Wang, X.; Zhang, Y.; Hu, X.; Ruan, J. Fertigation management for sustainable precision agriculture based on Internet of Things. J. Clean. Prod. 2020, 277, 124119. [CrossRef]

154. Nikolaou, G.; Neocleous, D.; Katsoulas, N.; Kittas, C. Irrigation of greenhouse crops. Horticulturae 2019, 5, 7. [CrossRef]

155. Benyezza, H.; Bouhedda, M.; Rebouh, S. Zoning irrigation smart system based on fuzzy control technology and IoT for water and energy saving. J. Clean. Prod. 2021, 302, 127001. [CrossRef]

156. Guo, X. Application of agricultural IoT technology based on 5 G network and FPGA. Microprocess. Microsyst. $2021,80,103597$. [CrossRef]

157. Hu, H.; Vocational, Y.; Chen, Z.; Science, I.; Wu, P.W.; Science, I. Internet of Things-Enabled Crop Growth Monitoring System for Smart Agriculture. Int. J. Agric. Environ. Inf. Syst. 2021, 12, 30-48. [CrossRef]

158. Said Mohamed, E.; Belal, A.A.; Kotb Abd-Elmabod, S.; El-Shirbeny, M.A.; Gad, A.; Zahran, M.B. Smart farming for improving agricultural management. Egypt. J. Remote Sens. Space Sci. 2021, in press. [CrossRef]

159. Wan-Soo, K.; Won-Suk, W.; Yong-Joo, K. A Review of the Applications of the Internet of Things (IoT) for Agricultural Automation. J. Biosyst. Eng. 2020, 45, 385-400. [CrossRef]

160. Hincapie, E.; Sánchez, J.; Carbonell, J. IoT Network Applied to Agriculture: Monitoring Stations for Irrigation Management in Soils Cultivated with Sugarcane. In Proceedings of the Advances in Information and Communication Technologies for Adapting Agriculture to Climate Change II, AACC 2018, Cali, Colombia, 21-23 November 2018; Springer: Cham, Switzerland, 2018; pp. 249-260. 
161. Cohen-Manrique, C.; Burbano-Bustos, A.; Salgado-Ordosgoitia, R.; Merlano-Porto, R. Irrigation control in ahuyama crops in Sincelejo, Sucre (Colombia) managed through the Internet of Things (IoT). Inf. Tecnol. 2020, 31, 79-88. [CrossRef]

162. Nuñez, J.; Fonthal, F.; Quezada, Y. Design and Implementation of WSN and IoT for Precision Agriculture in Tomato Crops. In Proceedings of the 2018 IEEE ANDESCON, Santiago de Cali, Colombia, 22-24 August 2018; pp. 1-5.

163. Tang, Y.; Dananjayan, S.; Hou, C.; Guo, Q.; Luo, S.; He, Y. A survey on the 5G network and its impact on agriculture: Challenges and opportunities. Comput. Electron. Agric. 2021, 180, 105895. [CrossRef]

164. Cama-Pinto, D.; Holgado-Terriza, J.A.; Damas-Hermoso, M.; Gómez-Mula, F.; Cama-Pinto, A. RadioWave Attenuation Measurement System Based on RSSI for Precision Agriculture: Application to Tomato Greenhouses. Inventions 2021, 6, 66. [CrossRef]

165. Selem, E.; Fatehy, M. E-Health applications over 5G networks: Challenges and state of the art. In Proceedings of the 2019 6th International Conference on Advanced Control Circuits and Systems (ACCS) \& 2019 5th International Conference on New Paradigms in Electronics \& information Technology (PEIT), Hurgada, Egypt, 17-20 November 2019; pp. 111-118.

166. Casquero Jiménez, A.; Pérez Martínez, J. 5G networks in eHealth services in Spain: Remote patient monitoring system. In Proceedings of the 2020 IEEE Engineering International Research Conference (EIRCON), Lima, Peru, 21-23 October 2020; pp. 20-23.

167. Emokpae, L.; Emokpae, R.; Lalouani, W.; Younis, M. Smart Multimodal Telehealth-IoT System for COVID-19 Patients. IEEE Pervasive Comput. 2021, 20, 73-80. [CrossRef]

168. Rokonuzzaman, M.; Hossain, I.M.; Islam, T.; Sarkar, P.P.; Islam, R.M.; Amin, N. Design and Implementation of Telehealth Device: Linking IoT Sensors to Cloud Networks. In Proceedings of the 2020 IEEE-EMBS Conference on Biomedical Engineering and Sciences (IECBES), Langkawi Island, Malaysia, 1-3 March 2021; pp. 281-285.

169. Gupta, N.; Juneja, P.K.; Sharma, S.; Garg, U. Future aspect of 5G-IoT architecture in smart healthcare system. In Proceedings of the 5th International Conference on Intelligent Computing and Control Systems (ICICCS), Madurai, India, 6-8 May 2021; pp. 406-411. [CrossRef]

170. Braeken, A.; Liyanage, M. Highly efficient key agreement for remote patient monitoring in MEC-enabled 5G networks. J. Supercomput. 2021, 77, 5562-5585. [CrossRef]

171. López, F.; Bonfante, M.C.; Gonzalez, I.; Muñoz, R. IoT and big data in public health: A case study in Colombia. In Protocols and Applications for the Industrial Internet of Things; IGI Global: Hershey, PA, USA, 2018; pp. 309-321.

172. Bolívar, N.; Cangrejo, L.; Salcedo, O. eHeart-BP, Prototype of the Internet of Things to Monitor Blood Pressure. In Proceedings of the 2019 IEEE/ACM International Conference on Connected Health: Applications, Systems and Engineering Technologies (CHASE), Arlington, VA, USA, 25-27 September 2019; pp. 58-63.

173. Hernández-Hernández, G.; Romero-Garavito, M.; Rodríguez-Manrique, J. The prototype of IoT Technological Infrastructure for Monitoring Vital Signs in Patients in an Emergency Department. In Proceedings of the LACCEI International Multi-Conference for Engineering, Education and Technology, Lima, Peru, 18-20 July 2018.

174. Huertas, T. Biomedical IoT Device for Self-Monitoring Applications. In Proceedings of the 7th Latin American Congress on Biomedical Engineering, CLAIB 2016, Bucaramanga, Colombia, 26-28 October 2016; pp. 357-360.

175. Haider, D.; Yang, X.; Hussain, Q. Post-surgical fall detection by exploiting the 5G C-Band technology for eHealth paradigm. Appl. Soft Comput. J. 2019, 81, 105537. [CrossRef]

176. Zhang, Y.; Chen, G.; Du, H.; Yuan, X.; Kadoch, M. Real-Time Remote Health Monitoring System Driven by 5G MEC-IoT. Electronics 2020, 9, 1753. [CrossRef]

177. Chowdhury, M.Z.; Hossan, T.; Shanjalal, M.; Hasan, K.; Jang, Y.M. A New 5G eHealth Architecture Based on Optical Camera Communication: An Overview, Prospects, and Applications. IEEE Consum. Electron. Mag. 2020, 9, 23-33. [CrossRef]

178. Artemenko, A.; Gmbh, R.B. Keynote: Advances and Challenges of Industrial IoT. In Proceedings of the PerIoT 2021, 5th International Workshop on Mobile and Pervasive Internet of Things, Kassel, Germany, 26 March 2021; p. 526.

179. Khatib, E.J.; Barco, R. Optimization of 5G Networks for Smart Logistics. Energies 2021, 14, 1758. [CrossRef]

180. Varsier, N.; Dufrène, L.; Dumay, M.; Lampin, Q.; Schwoerer, J. A 5G New Radio for Balanced and Mixed IoT Use Cases: Challenges and Key Enablers in FR1 Band. IEEE Commun. Mag. 2021, 59, 82-87. [CrossRef]

181. Varga, P.; Peto, J.; Franko, A.; Balla, D.; Haja, D.; Janky, F.; Soos, G.; Ficzere, D.; Maliosz, M.; Toka, L. 5G Support for Industrial Iot Applications-Challenges, Solutions, and Research Gaps. Sensors 2020, 20, 828. [CrossRef] [PubMed]

182. Eit Digital. Ericsson Define the Future of the Industrial IoT. Available online: https://www.eitdigital.eu/fileadmin/files/2017 /events/innovationdays/netherlands/Etienne_Scholl_-_Beyond_communication_the_digital_business_potential_of_5G.pdf (accessed on 15 October 2021).

183. Superintendencia de Industria y Comercio. The Internet of Things (IoT) Impacting the Logistics Sector. Available online: https://www.sic.gov.co/sites/default/files/files/pdf/Boletin_IoT(1).pdf (accessed on 9 March 2019).

184. Segura, D.; Khatib, E.; Munilla, J.; Barco, R. 5G Numerologies Assessment for URLLC in Industrial Communications. Sensors 2021, 21, 2489. [CrossRef] [PubMed]

185. You, X.; Zhang, C.; Tan, X.; Jin, S.; Wu, H. AI for 5G: Research directions and paradigms. Sci. China 2019, 62, 21301. [CrossRef]

186. Ministry of Environment. Deforestation in Meta, Caquetá and Guaviare Decreases by 30\% during the First Quarter of 2021. Available online: https:/ / www.minambiente.gov.co/index.php/noticias/5189-disminuye-en-un-30-porciento-la-deforestacionen-el-meta-caqueta-y-guaviare-durante-primer-trimestre-del-ano (accessed on 8 June 2021). 
187. AWS. Tackling Deforestation in Colombia with IoT at the 2019 Zoo Hackathon. Available online: https:/ / aws.amazon.com/es / blogs/publicsector/tackling-deforestation-colombia-zoo-hackathon/ (accessed on 2 May 2021).

188. Naveenraj, M.; Jeevabarathi, C.T.; Srinivasan, R. Iot based anti-poaching alarm system for trees in forest. Int. J. Innov. Technol. Explor. Eng. 2019, 8, 193-195.

189. Medellín, P. Deforestation by Fires in the Orinoquia and Amazonia Affect Air Quality in Bogota and Other Major Cities. Available online: http:/ /ieu.unal.edu.co/en/medios/noticias-del-ieu/item/la-deforestacion-por-incendios-en-la-orinoquiay-la-amazonia-afectan-la-calidad-del-aire-de-bogota-y-otras-grandes-ciudades (accessed on 8 June 2021).

190. Sahal, R.; Alsamhi, S.; Breslin, J.; Intizar Ali, M. Industry 4.0 towards Forestry 4.0: Fire Detection Use Case. Sensors 2021, $21,694$. [CrossRef]

191. Khan, I.; Ahmad, S.; Kim, D.H. A Task Orchestration Approach for Efficient Mountain Fire Detection Based on Microservice and Predictive Analysis in IoT Environment. J. Intell. Fuzzy Syst. 2021, 40, 5681-5696. [CrossRef]

192. Nguyen-Anh, T.; Le-Trung, Q. Prediction of Forest Fire Risk to Trigger IoTs Reconfiguration Action. In Proceedings of the 2020 7th NAFOSTED Conference on Information and Computer Science (NICS), Ho Chi Minh, Vietnam, 26-27 November 2020; pp. 19-24.

193. Das, P.; Ghosh, S.; Chatterjee, S.; De, S. Energy Harvesting-enabled 5G Advanced Air Pollution Monitoring Device. In Proceedings of the 2020 IEEE 3rd 5G World Forum, (5GWF 2020), Bangalore, India, 10-12 September 2020; pp. 218-223. [CrossRef] 